Article

\title{
Evidence-Based Resilience Management for Supply Chain Sustainability: An Interpretive Structural Modelling Approach
}

\author{
Nina Shin ${ }^{1}$ and Sangwook Park ${ }^{2, *}$ \\ 1 College of Business Administration, Sejong University, Seoul 05006, Korea; ninashin@sejong.ac.kr \\ 2 College of Business Administration, Seoul National University, Seoul 08826, Korea \\ * Correspondence: sangpark@snu.ac.kr; Tel.: +82-2-880-5762
}

Received: 13 December 2018; Accepted: 15 January 2019; Published: 17 January 2019

check for updates

\begin{abstract}
The purpose of this study is to systematically identify and design improvement planning for supply chain resilience (SCRES) for a higher level of sustainability and a competitive advantage. Literature-based interpretive structural modelling (ISM), a pairing of the systematic literature review (SLR) and ISM approaches, is proposed for investigating and identifying a set of key performance measures of resilience for supply chain (SC) management. In line with previous research, we identified and updated 13 key SC capabilities out of 24 as core performance measures of supply network resilience. Furthermore, our findings categorised each capability and element into one of four types of influential power variables (drivers, dependent, autonomous, or linkage). This study (i) lends support to and updates previous research that examined the core capabilities of SCRES and (ii) provides complementary classifications for the influential powers of SCRES capabilities and elements. The literature indicates that there has been no research that has integrated SLR as a basis to ISM for an effective way to utilize existing studies for increasing awareness and developing managerial guidelines to achieve SCRES.
\end{abstract}

Keywords: Supply chain resilience; sustainability; systematic literature review; interpretive structural modelling

\section{Introduction}

Supply chain resilience (SCRES) differs from vulnerability or disruption management, as it heavily relies on both internal and external operational capabilities in addressing both expected and unexpected events [1-4]. Although the definitions of resilience vary according to the context (i.e., physical, ecological, socio-ecological, psychological, disaster management, organisational, and engineering), resilience in supply chain (SC) management is defined as the ability of a firm to cope with the consequences of unavoidable events in order to return to its original operations or move to a new, more desirable state after being disturbed' [4,5]. SCRES is also primarily represented as the state of the art in which a firm absorbs, adapts to, and restores itself from any disruptive event, ranging from a minor shipment delay to a major earthquake [6]. Further, SCRES has gained attention from both researchers and practitioners, primarily due to two main reasons: (1) The increase in network complexity driven by global transactions and (2) an over-reliance on technology-driven operational solutions. An increase in the supply network's complexity considerably raises the cumulative risk level contributed by the participating members of the network [7-9]. Moreover, the frequency of disruptions and level of risk exposure are mostly environmentally driven, and, therefore, are uncontrollable from a single firm's perspective [10].

Unfortunately, the multitude of studies on SCRES is unequivocal regarding the applicability of SCRES measures. There has been a lack of guidance specifically for business practitioners in 
understanding the interrelationship among SC capabilities and their usability and implications from a managerial perspective.

Existing SC capabilities or elements are an essential asset in preparing for potential business threats and opportunities. Notably, Pettit et al. [3] developed an SCRES framework based on the following seven categories of vulnerabilities: Turbulence, deliberate threats, external pressures, resource limits, sensitivity, connectivity, and supplier-customer disruptions. The authors stated that such vulnerabilities should be managed by the management control tower, which ultimately forms SC capabilities: Flexible sourcing and order fulfilment, capacity, efficiency, visibility, adaptability, anticipation, recovery, dispersion, collaboration, organisation, market position, security, and financial strength. Pettit et al. [11] proposed a measurement tool—called Supply Chain Resilience Assessment and Management-which emphasizes the need for a balance between vulnerability and capabilities to manage them effectively and better than competitors. Since then, various empirical investigations of SC capabilities in resilience contexts have proliferated. Jüttner and Maklan [5] found that risk management strategies significantly affect SC members' levels of vulnerability (i.e., revenues, costs, and lead time/agility) and resilience (i.e., flexibility, velocity, visibility, and collaboration). Bhamra et al. [12] stated that small and medium-sized enterprises can achieve resilience by adapting to risks based on their existing capabilities and resource availability. Hohenstein et al.'s [13] systematic review of 67 peer-reviewed articles from 2003 to 2013 identified 36 SCRES elements, among which flexibility, redundancy, collaboration, visibility, agility, and multiple sourcing were the top six in terms of the number of appearances in various studies. Recently, Ali et al. [6] identified 13 essential resilience elements and 84 managerial practices based on 103 research articles for enhancing firms' capabilities in anticipating, adapting and responding to, and recovering and learning from disruptive events. However, the existing literature has hitherto focused on the comprehensive framework or theory-driven investigation of SCRES.

\subsection{Motivation and Contribution}

Recent studies on SCRES and sustainability have investigated various aspects ranging from identifying enablers of and barriers to SCRES elements (i.e., how to create and how to achieve SCRES?) [14], an appropriate SCRES definition and essential SCRES elements (i.e., what is SCRES, and how to proactively and reactively prepare for an ex-ante/ex-post disruption?) [13], theoretical foundations of SCRES (i.e., how to continuously manage risk migration in addition to specific and isolated threats?) [15], and a conceptual mapping framework of SCRES (i.e., what are different features of SCRES?) [6].

Traditional SC capabilities and elements are examined to discover how to effectively prepare, control, and recover from disruption. As a result, many studies identified SCRES as an essential strategic capability of a firm to survive and sustain in competitive yet unpredictable events $[2,6,16]$. In this regard, SCRES practices improve a traditional SC network system and its sustainability by considering network relationships, environmental issues, and obtainable competitive advantages.

In this sense, this study raises some questions:

1. What are key performance measures for SCRES practices?

2. How can practitioners systematically identify and design SCRES improvement planning for a higher level of sustainability and a competitive advantage?

The specific objectives of the present study are:

1. To identify and highlight key SC elements for the operationalization of SCRES practices that are both theoretically and empirically appropriate;

2. To understand the interdependent and influential relationships among these elements, and to provide managerial guidance in achieving SCRES success.

To fulfil these objectives, this study adopts a two-phase methodology, which integrates (i) a systematic literature review (SLR) to identify core SCRES elements, and (ii) a graph-based interpretive 
structural modelling (ISM) to visually identify interdependent and influential powers of SCRES elements. The SLR approach is valued as a decision-making methodology because it provides a rigorous and transparent means to manage and synthesize the diversity of knowledge, create new knowledge, and generalise findings [17]. The approach has been applied to several aspects of SCRES and sustainability $[10,18,19]$. Likewise, ISM has been applied for identifying a map of the relationships among practices and performance measures in resilient and sustainable SC management $[20,21]$. ISM plays a complementary role to SLR as it is useful in finding structural relationships among a large number of elements under investigation. ISM visually transforms complex relationships into a relationship hierarchy presenting varying levels of depth, ultimately assisting managers to observe the elements' dependent or driving powers. Therefore, we choose an SLR-based ISM approach for examining the interdependent relationships among SCRES elements and ultimately propose the evidence-based ISM approach that can be utilized for minimizing the gap between researchers' and practitioners' understanding.

This study integrates the SLR and ISM approaches to identify a 'core hierarchy' based on a systematic procedure. The finding reveals the interactions and the resulting interdependent structural relationships among SCRES capabilities and elements. The proposed evidence-based approach contributes to the stream of literature on SCRES by lending (i) support and updates to previous research that examined the core SCRES capabilities, and (ii) complementary classifications of influential powers of SCRES capabilities and elements. To the best of our knowledge, the literature indicates that there has been no study that has systematically analysed the interaction of capabilities of SCRES management based on the existing number of research articles that have already been proven to be theoretically and empirically sound through publications in peer-reviewed journals.

\subsection{Organisation of the Paper}

The remainder of this paper proceeds as follows: Section 2 presents the proposed research design and methodology, and the application of the proposed approach is presented in Section 3. The results and discussion, including a comparison with the existing literature, are provided in Section 4. Finally, theoretical and managerial implications, as well as this study's conclusion, limitations, and opportunities for future research, are given in Section 5.

\section{Research Methodology: An Evidence-Based Methodology Approach}

This study proposes an evidence-based ISM, consisting of two different approaches-an SLR and ISM-as a useful tool in systematically gaining knowledge about a given topic and organising the findings. Management reviews can often be narrative and be potentially exposed to the inclusion of biases, thereby lacking critical assessment [22]. As an alternative, evidence-based approach, initially developed by the British central government in the 1980s primary focusing on 'three $E^{\prime}$ initiatives (economy, efficiency, effectiveness), it has made an impact in the healthcare industry for its "replicable, scientific, and transparent process, which detailed a technology, that aims to minimize bias through extensive literature searches" [22]. The proposed evidence-based approach in this study is unique as it extends the results of SLR by performing the ISM part of the analysis. Figure 1 demonstrates the step-by-step procedure of the proposed approach, which aims to maintain the core value of SLR while heightening its value by increasing the objectivity of the results derived from quantitative analysis to encourage other researchers to replicate the process. Recently, the SLR approach has gained a credible reputation as a methodology for a strict 'set of guidelines, adopting a replicable, scientific, and transparent process' [6]. ISM has also achieved reliability as an effective methodology for identifying both interactivities and hierarchical structures based on an expert consensus [23]. As existing studies are based on theoretical and empirical evidence, they are deemed to represent a larger number of experts compared to the size of the panel of experts that would have been adopted to construct the initial matrix or hypotheses. Thus, the relevant literature collection was processed 
through a rigorous SLR and was funnelled through the ISM process to extract, synthesize, and visually demonstrate the summary of the findings.

\subsection{SLR Approach}

The SLR approach provides a strong basis for knowledge advancement, theory development, and the discovery of new research streams [24]. The most popular research protocol proposed by Tranfield et al. [22] and Kitchenham [25], which contains five different phases, is adopted: (i) Identification of research, (ii) selection of studies, (iii) study quality assessment, (iv) data extraction and monitoring progress, and ( $v$ ) data synthesis. Table 1 replicates the research protocol as recommended by Alexander et al. [26] and describes how SLR is utilized to advance the understanding and interpretation of existing findings, which include over 100 articles published between 2003 and 2017, relative to the SC capabilities and elements that lead to resilient management outcomes.

Table 1. Systematic review protocol.

\begin{tabular}{|c|c|}
\hline Review Protocol & Description \\
\hline \multicolumn{2}{|c|}{ Identification of research } \\
\hline Databases & $\begin{array}{l}\text { Automatic search through ScienceDirect and Web of Knowledge for their relevancy } \\
\text { and availability of high impact factor publications [27]. } \\
\text { Manual search though Google Scholar online databases: The manual search aims to } \\
\text { ensure the article database's comprehensiveness and relative completeness. Backward } \\
\text { and forward search methods [25] were respectively used to manually search for } \\
\text { citations from the selected studies, and to find the studies that cite the selected primary } \\
\text { studies through the Google Scholar search engine. }\end{array}$ \\
\hline Publication type & Review articles, research articles, and case studies. \\
\hline Language & English-only due to lack of translation capacity. \\
\hline Date range & Published within selected period of time (2003-2017). \\
\hline Search fields & Search terms were applied to Titles, Abstracts, and Keywords only. \\
\hline Search terms & $\begin{array}{l}\text { The main keywords were identified based on the research question from this review: } \\
\text { ('supply chain capability' AND 'supply chain resilience') OR ('supply chain capability' } \\
\text { AND 'resilience'). }\end{array}$ \\
\hline \multicolumn{2}{|l|}{ Selection of studies } \\
\hline $\begin{array}{l}\text { Exclusion criteria \#1: } \\
\text { Semantic relevance }\end{array}$ & $\begin{array}{l}\text { The primary exclusion criterion is its relevance to the research topic, SC capabilities } \\
\text { and SC resilience. As noted by Hohenstein et al. [13], the terminologies related to SC } \\
\text { resilience elements are inconsistent, and the element is also referred to as antecedents, } \\
\text { enhancers, competencies, capabilities, and attributes. To remain within the scope of } \\
\text { this study, we identified capabilities and elements as a set of operational variables that } \\
\text { SC managers can improve on through their firms' routines, procedures, and process } \\
\text { applications [28], and we only included the studies specifically related to SC } \\
\text { capabilities and elements in a resilience context (henceforth, SC capabilities and SC } \\
\text { elements are used interchangeably). }\end{array}$ \\
\hline $\begin{array}{l}\text { Exclusion criteria \#2: } \\
\text { Relevance to the } \\
\text { research question }\end{array}$ & $\begin{array}{l}\text { Secondary exclusion criterion is relevance to the research question. Full texts were } \\
\text { reviewed to determine the relevance to SCRES-driven research objectives. For example, } \\
\text { studies that do not primarily aim to identify SCRES conceptual definition, } \\
\text { enablers/barriers of SCRES, SCRES driven performance, and SCRES-based mitigation } \\
\text { strategy are excluded. }\end{array}$ \\
\hline
\end{tabular}

Quality assessment process. Based on the initially collected database of 362 articles, the studies relevant to the research question were determined through selection and a quality assessment. First, studies were screened for availability of the full text in English, publication dates between 2003 and 2017, and an SC management research domain. Articles were then reviewed to assess each study's quality based on three criteria: (i) The topic's relevance to SCRES, (ii) the availability of a data collection, methodology, and analysis description, and (iii) the availability of a theoretical and/or empirical basis 
for the findings. The application of quality assessment is considered critical as the quality instruments driven database can enrich confidence in the credibility of the selected studies [27]. Consequently, 149 research articles were selected.
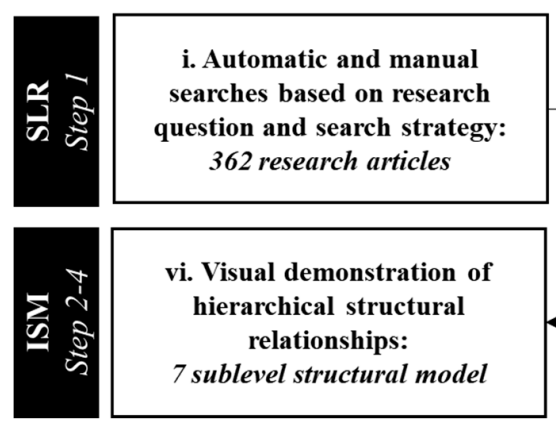

vi. Visual demonstration of hierarchical structural relationships:

7 sublevel structural model

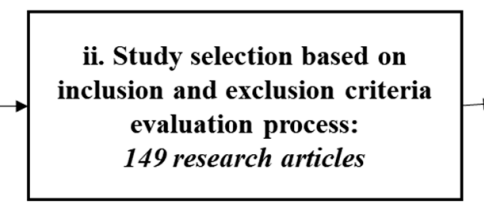

v. Finalization of interrelationship based on the initial/final reachability matrix: 533 relationships

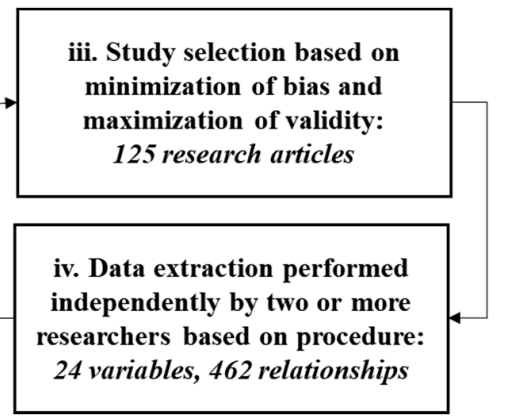

iii. Study selection based on minimization of bias and aximization of validity: 125 research articles

Figure 1. Steps for conducting evidence-based interpretive structural modelling (ISM).

Data extraction. As noted by Hohenstein et al. [13], the terminologies related to SCRES elements are inconsistent, and the element are also referred to as antecedents, enhancers, competencies, capabilities, and attributes. To remain within the scope of this study, we identified capabilities and elements as a set of operational variables that SC managers can improve on through their firms' routines, procedures, and process applications [28], and we extracted the studies specifically related to SC capabilities and elements in a resilience context (henceforth, SC capabilities and SC elements are used interchangeably). This process was performed by reading each study carefully, and extracting the related data using Mendeley and Microsoft Excel spreadsheets. The inconsistent definition and usability of SC elements exist among studies (i.e., some refer to agility and flexibility with identical meaning, while others refer to them as distinctive capabilities). Thus, we created definitions and relevant keywords for each element and combined elements based on the similarity of definition and the used contexts. A set of 125 research articles was ultimately identified for the final investigation, collectively producing 24 sets of SC capabilities and elements: $\zeta_{1}$ : SC Network Design, $\zeta_{2}$ : Flexibility, $\zeta_{3}$ : Redundancy, $\zeta_{4}$ : Visibility, $\zeta_{5}$ : Collaboration, $\zeta_{6}$ : Agility and Responsiveness, $\zeta_{7}$ : Alertness, Anticipation, and Awareness, $\zeta_{8}$ : Information Technology and Information Sharing, $\zeta_{9}$ : Robustness, $\zeta_{10}$ : SCRM Culture, $\zeta_{11}$ : Security, $\zeta_{12}$ : Knowledge Management, $\zeta_{13}$ : SC Continuity and Contingency Planning, $\zeta_{14}$ : Velocity, $\zeta_{15}$ : Coordination, $\zeta_{16}$ : Integration, $\zeta_{17}$ : Adaptability, $\zeta_{18}$ : Market Position, $\zeta_{19}$ : Risk Control, $\zeta_{20}$ : Alignment, $\zeta_{21}$ : Public-Private Partnership, $\zeta_{22}$ : Efficiency, $\zeta_{23}$ : Trust, $\zeta_{24}$ : Sustainability.

\subsection{ISM Approach}

ISM is effective as it uses a structural representation to identify and synthesize the different levels of a given problem. This modelling approach was initially created to configure a sequence of contextual relationships or elements, and to assist decision-makers in understanding complex systems, thereby creating appropriate action plans [29]. The ISM methodology has gained popularity for two different research objectives. First, for an identification of order and direction in complex process systems, Wu et al. [30] clarified and established levels of problem structures and prioritized factors relative to the operational flow in an offshore pipeline project. Faisal et al. [31] identified six levels in an SC's information risk mitigation process and analysed them based on four different categories: Autonomous, dependent, linkage, and independent enablers. Second, as a way to delineate critical practices or elements that are superior in determining other ones, Govindan et al. [32] identified critical lean, green, and resilient practices that top managements should focus on to improve SC performance in an automotive context. Diabat et al. [33] defined five risk types involved in a food SC and recommended corresponding risk-mitigation strategies. As this study intends to provide a set of decisions for controlling and improving firm resilience based on a visual representation 
of key SC capabilities and elements, the ISM approach is deemed most appropriate to synthesize SLR-driven data.

The fundamental ISM process involves preparing an initial matrix (structural-self interaction matrix, SSIM), consisting of elements of interest. A reachability matrix is then formed based on experts' evaluation of the elements' relationship (i.e., whether variable A affects variable B). Subsequently, the final matrix, displaying the final interactivity relationships through a hierarchical structure, is created. For brevity, these steps are described in the next section.

\subsection{Evidence-based ISM Approach}

The goal of the proposed method is to expand the application level of ISM based on the existing SLR to decompose a complex bundle of SC elements into sub-systems for a multilevel structural model. The detailed step-by-step procedure of this application of the graph-theoretic approach is as follows:

Step 1: Identifying the relevant elements. To begin, a database of articles relevant to the specific topic (in this study, SCRES) are selected through the SLR process described in Section 2.1. During this stage, the existing research articles are collected and evaluated to establish a comprehensive set of elements that have been theoretically and empirically verified to have a significant impact on the resilience outcome.

Step 2: Identifying the influential relationships (SSIM). The directed relationships are established (rather than hypothesized, as we assume that the existing studies have already verified the hypothetical relationships). The meaning of 'directed' refers to the direction of the relationship, if one exists, between any two SC elements: From $i$ to $j$, from $j$ to $i$, or in both directions). Based on the identified contextual relation, an SSIM is developed based on pairwise comparisons of the SC elements.

Step 3: Forming an initial and final reachability matrix. Based on the developed SSIM, first, an initial binary reachability matrix is formed to reflect the directed relationships between all SC elements (an element is 1 if the relationship exists, and 0 otherwise). If more than one research article identified the directed relationship, then the corresponding number of articles is used instead of a 1 . Last, the final reachability matrix is developed to include transitivity characteristics (i.e., if $i$ affects $j$, and $j$ affects $k$, then $i$ affects $k: T$ if a transitivity relationship exists, and 0 otherwise). In summary, the reachability matrix answers the question: Can we 'reach' element $j$ by starting at element $i$ ? where 'reach' includes both a direct and indirection relationship from $i$ to $j$ [32].

This stage is distinctly different from the conventional ISM approach. The conventional ISM approach would initially build 'hypothetical relationships', which are to be evaluated by the practitioners. Because we are employing existing studies based on well-established theoretical and empirical measures that are either theoretically or practically confirmed by real-world cases, we can develop and finalize reachability simultaneously. The approach in which we interpret the existing literature to create a 'direct relationship' is based on either verified hypothetical relationships or on authors' statements in establishing potential hypothetical relationships. For example, Roberta-Pereira et al. [14] noted that 'flexibility seems to be an advantageous way of increasing agility and resilience in the end'. We would translate this contextual evidence as a confirmation that a direct relationship exists between 'flexibility' and 'agility'.

Step 4: Analysing the hierarchical levels of SC capabilities. The final reachability matrix can now be decomposed as a structural model. This systematic process provides the grouping of various SC elements into different sub-systems or multi-levels depending on their directed relationships. As a result, the multilevel ISM model is illustrated to show the interrelationships among elements more clearly.

\section{Application of the Proposed Approach}

This section describes the application of the proposed evidence-based ISM approach (Figure 1).

Step 1: Identifying the relevant elements. The SLR approach was used to reveal 24 SC elements that enable and contribute to SCRES: SC Network Design; Flexibility; Redundancy; Visibility; Collaboration; 
Agility and Responsiveness; Alertness, Awareness, and Alertness; Information Technology and Information Sharing; Robustness; SC Risk Management (SCRM) culture, Security, Knowledge Management, SC Continuity, and Contingency Planning; Velocity; Coordination; Integration and Connectivity; Adaptability; Market Position; Risk Control; Alignment; Public-Private Partnership (PPP); Efficiency; Trust; and, Sustainability.

Step 2: Identifying the potential influential relationships (SSIM). The collection of studies is reviewed for specific statements or findings that address relationships among SC elements for evidence of interrelationships, as in the examples in Table 2.

Table 2. Examples of identified literature evidence indicating a 'direct' relationship.

\begin{tabular}{lc}
\multicolumn{1}{c}{ Literature Evidence } & $\begin{array}{c}\text { Indication of Direct Relationship } \\
(i \rightarrow j)\end{array}$ \\
\hline $\begin{array}{l}\text { "Visibility, velocity, and flexibility together are sometimes } \\
\text { captured under agility." [5] }\end{array}$ & 'Visibility', 'Velocity', 'Flexibility' affects 'Agility" \\
\hline $\begin{array}{l}\text { "Collaboration is related to visibility in the sense that it } \\
\text { includes an organization's willingness to share even } \\
\text { sensitive risk and risk event-related information." [34] }\end{array}$ & 'Collaboration' affects 'Visibility' \\
\hline $\begin{array}{l}\text { "Control (coordination of activities), coherence, and } \\
\text { connectedness are key components of resilience's } \\
\text { efficient response." [2] }\end{array}$ & 'Coordination' affects 'Responsiveness' \\
\hline $\begin{array}{l}\text { "Supply chain connectivity and information sharing } \\
\text { resources lead to a supply chain visibility capability." [35] }\end{array}$ & 'Integration' affects 'Visibility' \\
\hline
\end{tabular}

Upon the initial reading and collection of literature evidence of direct relationships, three academics, whose main research interest is SC management, reviewed and eliminated the evidence that does not seem to represent a direct relationship appropriately. For each research article, all the variables were identified and evaluated based on the existence of the 'leads to' or 'affects' type of contextual relationship between any two variables ( $i$ and $j$ ). In other words, if one variable ( $i$ ) was contextually stated to lead to another variable ( $j$ ), the final interrelationship was recorded. This process was conducted for both $i$ on $j$ and vice versa, for a total of $24 \times 24$ matrix elements. The identified relationships among SC elements are summarized in Table 3.

Step 3a: Forming an initial reachability matrix. The directed relationships between the SC elements, any pair of variables $i$ and $j$, are recorded in the initial reachability matrix as the number of articles providing evidence of an existing relationship (Table 4); otherwise a zero is recorded.

Step $3 b$ : Finalizing the reachability matrix. To include significant transitive relationships (i.e., if $i$ affects $j$ and $j$ affects $k$, then $i$ affects $k$ ), such relationships based on the previously formed direct matrix are identified as ' $T$ '. Table 5 illustrates the final reachability matrix of SC elements. Moreover, the quantitative representation of influential powers is summarized as the driving (DRV.) and dependence (DEP.) sums in the last column and last row, respectively.

Step 4: Analysing the hierarchical levels of SC elements. Based on the reachability, antecedents, and intersection elements, SC elements are sorted in a number of iterations to form relationships of hierarchical influence (Table 6). If the reachability set and intersection set are the same, then the variables are positioned at the top of the ISM hierarchy, signifying that no other primary element can be reached above this Level I group. Among the 24 SC capabilities, 13 are identified as the Level I group, as no other primary element can be reached above these elements. 
Table 3. The interrelationship of Supply Chain (SC) elements based on the literature review.

\begin{tabular}{|c|c|c|c|c|c|c|c|c|c|c|c|c|c|c|c|c|c|c|c|c|c|c|c|c|}
\hline & $\zeta_{1}$ & $\zeta_{2}$ & $\zeta_{3}$ & $\zeta_{4}$ & $\zeta_{5}$ & $\gamma_{6}$ & $\zeta_{7}$ & $\zeta_{8}$ & $\bar{\zeta}_{9}$ & $\zeta_{10}$ & $\zeta_{11}$ & $\zeta_{12}$ & $\zeta_{13}$ & $\zeta_{14}$ & $\zeta_{15}$ & $\zeta_{16}$ & $\zeta_{17}$ & $\zeta_{18}$ & $\zeta_{19}$ & $\zeta_{20}$ & $\zeta_{21}$ & $\zeta_{22}$ & $\zeta_{23}$ & $\zeta_{24}$ \\
\hline$\zeta_{1}$ & & & & [14] & $\begin{array}{l}\text { [13], } \\
{[36]}\end{array}$ & $\begin{array}{l}\text { [37], } \\
\text { [13], }\end{array}$ & & & & & & & [36] & & & & [36] & & [38] & & & $\begin{array}{l}{[39],} \\
{[40]}\end{array}$ & & \\
\hline$\zeta_{2}$ & $\begin{array}{l}\text { [41], } \\
{[42],} \\
{[43]}\end{array}$ & & $\begin{array}{c}{[44],} \\
{[5],} \\
{[45],} \\
{[46],} \\
{[47]}\end{array}$ & & & $\begin{array}{c}{[48],} \\
{[49],} \\
{[4],} \\
{[50],} \\
{[51],} \\
{[13],} \\
{[5],} \\
{[52],} \\
{[53],} \\
{[54],} \\
{[55],} \\
{[45],} \\
{[56],} \\
{[57],} \\
{[14],} \\
{[58],} \\
{[46],} \\
{[14],} \\
{[59],} \\
{[60],} \\
{[61],} \\
{[62]}\end{array}$ & $\begin{array}{c}\text { [5], } \\
{[63],} \\
{[46]}\end{array}$ & & $\begin{array}{l}\text { [35], } \\
{[52],}\end{array}$ & [64] & & [44] & & $\begin{array}{r}{[5],} \\
{[65]}\end{array}$ & & [66] & & [51] & $\begin{array}{l}\text { [67], } \\
{[38],} \\
{[68],} \\
{[64]}\end{array}$ & & & $\begin{array}{l}\text { [69], } \\
{[57],} \\
{[58]}\end{array}$ & [44] & \\
\hline$\zeta_{3}$ & [51] & $\begin{array}{c}{[70],} \\
{[44],} \\
{[71],} \\
{[1]}\end{array}$ & & & & [14] & [46] & & [62] & & & & [64] & [14] & & [51] & [51] & & $\begin{array}{l}\text { [66], } \\
\text { [43] }\end{array}$ & & & & [44] & \\
\hline$\zeta_{4}$ & $\begin{array}{l}{[72],} \\
{[44]}\end{array}$ & [65] & & & $\begin{array}{c}\text { [73], } \\
{[5]}\end{array}$ & $\begin{array}{c}{[48],} \\
{[4],} \\
{[13],} \\
{[5],} \\
{[65],} \\
{[74],} \\
{[47],} \\
{[43]} \\
{[75],} \\
{[61]}\end{array}$ & $\begin{array}{l}{[76],} \\
{[63],} \\
{[77],} \\
{[14],} \\
{[78]}\end{array}$ & $\begin{array}{l}\text { [35], } \\
{[79]}\end{array}$ & [72] & $\begin{array}{l}\text { [51], } \\
{[5],}\end{array}$ & & $\begin{array}{l}{[5],} \\
{[8]}\end{array}$ & [35] & [65] & & $\begin{array}{c}\text { [35], } \\
{[3]}\end{array}$ & & & $\begin{array}{l}\text { [73], } \\
{[80]}\end{array}$ & & & & [44] & \\
\hline
\end{tabular}


Table 3. Cont.

\begin{tabular}{|c|c|c|c|c|c|c|c|c|c|c|c|c|c|c|c|c|c|c|c|c|c|c|c|c|}
\hline & $\zeta_{1}$ & $\zeta_{2}$ & $\zeta_{3}$ & $\zeta_{4}$ & $\zeta_{5}$ & $\zeta_{6}$ & $\zeta_{7}$ & $\zeta_{8}$ & $\overline{\zeta_{9}}$ & $\zeta_{10}$ & $\zeta_{11}$ & $\zeta_{12}$ & $\zeta_{13}$ & $\zeta_{14}$ & $\zeta_{15}$ & $\zeta_{16}$ & $\zeta_{17}$ & $\zeta_{18}$ & $\zeta_{19}$ & $\zeta_{20}$ & $\zeta_{21}$ & $\zeta_{22}$ & $\zeta_{23}$ & $\zeta_{24}$ \\
\hline$\zeta_{5}$ & [81] & $\begin{array}{c}{[49],} \\
{[82],} \\
{[83],} \\
{[5],} \\
{[54],} \\
{[65],} \\
{[84],} \\
{[85],} \\
{[86],} \\
{[59],} \\
{[78],}\end{array}$ & & $\begin{array}{l}\text { [4], } \\
{[51],} \\
{[80],} \\
{[84]}\end{array}$ & & $\begin{array}{l}\text { [81], } \\
{[57]}\end{array}$ & $\begin{array}{l}{[88],} \\
{[81],}\end{array}$ & $\begin{array}{l}\text { [84], } \\
{[78]}\end{array}$ & $\begin{array}{l}{[77],} \\
{[59]}\end{array}$ & $\begin{array}{l}\text { [35], } \\
\text { [81] }\end{array}$ & $\begin{array}{l}\text { [88], } \\
{[81],} \\
{[89]}\end{array}$ & & [81] & $\begin{array}{l}\text { [54], } \\
{[65],} \\
{[84]}\end{array}$ & 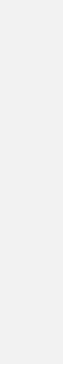 & $\begin{array}{c}{[81],} \\
{[3]}\end{array}$ & [81] & [81] & $\begin{array}{c}{[66],} \\
{[4],} \\
{[36],} \\
{[14],} \\
{[47],} \\
{[80],} \\
{[90]}\end{array}$ & [5] & [11] & $\begin{array}{l}{[83],} \\
{[91],} \\
{[92],} \\
{[59]}\end{array}$ & & \\
\hline$\zeta_{6}$ & & $\begin{array}{l}{[93],} \\
{[40]}\end{array}$ & & & [4], & & $\begin{array}{l}\text { [4], } \\
{[40]} \\
{[46]}\end{array}$ & [14] & [4] & [4] & & [4] & [94] & & & [61] & [4] & & $\begin{array}{c}\text { [4], } \\
{[95],} \\
{[38]}\end{array}$ & [38] & [2] & & & \\
\hline$\zeta_{7}$ & & $\begin{array}{l}\text { [87], } \\
{[64]}\end{array}$ & & $\begin{array}{l}{[81],} \\
{[44],} \\
{[77]}\end{array}$ & [81] & $\begin{array}{l}\text { [63], } \\
{[74]}\end{array}$ & & [74] & [74] & [74] & & & & [74] & & [74] & $\begin{array}{l}\text { [81], } \\
\text { [74] }\end{array}$ & [81] & [47] & & & [81] & & \\
\hline$\zeta_{8}$ & & $\begin{array}{l}{[49],} \\
{[28],} \\
{[38],} \\
{[54],} \\
{[84],} \\
{[85],} \\
{[86],} \\
{[59],} \\
{[96],} \\
{[97]}\end{array}$ & & $\begin{array}{l}{[98],} \\
{[99],} \\
{[35]} \\
{[100],} \\
{[72],} \\
{[91],} \\
{[76],} \\
{[44],} \\
{[5],} \\
{[54],} \\
{[14],} \\
{[59],} \\
{[78],} \\
{[101]} \\
{[96],} \\
{[102]}\end{array}$ & $\begin{array}{l}{[99],} \\
{[28],} \\
{[70],} \\
{[92],} \\
{[85],} \\
{[78]}\end{array}$ & $\begin{array}{l}{[69],} \\
{[70],} \\
{[57],} \\
{[14],} \\
{[60],} \\
{[96]}\end{array}$ & $\begin{array}{l}\text { [99], } \\
{[103],} \\
{[75]}\end{array}$ & & $\begin{array}{l}{[40],} \\
{[102]}\end{array}$ & $\begin{array}{l}{[103],} \\
{[96]}\end{array}$ & & & $\begin{array}{l}{[81],} \\
{[74]}\end{array}$ & [84] & {$[80]$} & & & & $\begin{array}{l}{[40],} \\
{[47],} \\
{[80]}\end{array}$ & & [96] & $\begin{array}{l}{[99],} \\
{[28],} \\
{[83],} \\
{[103],} \\
{[92]}\end{array}$ & [104] & \\
\hline$\zeta_{9}$ & $\begin{array}{l}\text { [73], } \\
{[72]}\end{array}$ & $\begin{array}{c}{[105],} \\
{[5],} \\
{[58],} \\
{[80],} \\
{[59]}\end{array}$ & [62] & [35] & $\begin{array}{l}\text { [35], } \\
\text { [101] }\end{array}$ & $\begin{array}{l}{[106],} \\
{[57],} \\
{[60],} \\
{[101],} \\
{[61]}\end{array}$ & [61] & & & [101] & [101] & [101] & [101] & [107] & & [61] & [101] & [62] & $\begin{array}{l}{[67],} \\
{[95],} \\
{[101],} \\
{[62]}\end{array}$ & & & $\begin{array}{l}{[71],} \\
{[43]}\end{array}$ & & \\
\hline
\end{tabular}


Table 3. Cont

\begin{tabular}{|c|c|c|c|c|c|c|c|c|c|c|c|c|c|c|c|c|c|c|c|c|c|c|c|c|}
\hline & $\zeta_{1}$ & $\zeta_{2}$ & $\zeta_{3}$ & $\zeta_{4}$ & $\zeta_{5}$ & $\overline{\zeta_{6}}$ & $\zeta_{7}$ & $\zeta_{8}$ & $\zeta_{9}$ & $\zeta_{10}$ & $\zeta_{11}$ & $\zeta_{12}$ & $\zeta_{13}$ & $\zeta_{14}$ & $\zeta_{15}$ & $\bar{\zeta}_{16}$ & $\zeta_{17}$ & $\zeta_{18}$ & $\zeta_{19}$ & $\zeta_{20}$ & $\zeta_{21}$ & $\zeta_{22}$ & $\zeta_{23}$ & $\zeta_{24}$ \\
\hline$\zeta_{10}$ & [51] & $\begin{array}{l}{[105],} \\
{[86]}\end{array}$ & & [108] & $\begin{array}{l}{[37],} \\
{[72],} \\
{[91],} \\
{[44],} \\
{[109],} \\
{[110]}\end{array}$ & $\begin{array}{l}{[57],} \\
{[58]}\end{array}$ & & [108] & [58] & & $\begin{array}{l}\text { [67], } \\
\text { [35], } \\
{[111]}\end{array}$ & & $\begin{array}{c}{[37],} \\
{[4],} \\
{[47],} \\
{[111],} \\
{[101]}\end{array}$ & & & & & & & & & $\begin{array}{l}\text { [57], } \\
\text { [80] }\end{array}$ & & \\
\hline$\zeta_{11}$ & & & & [89] & & & & $\begin{array}{l}{[88],} \\
{[89]}\end{array}$ & & [41] & & & & & & & & & & & & & $\begin{array}{l}\text { [41], } \\
{[89]}\end{array}$ & \\
\hline$\zeta_{12}$ & & & & [84] & [13] & & & & & & & & & & [84] & & & & & & & & & \\
\hline$\zeta_{13}$ & & [112] & [13] & & [113] & [2] & {$[4]$} & & & [4] & [41] & & & & & & [8] & & $\begin{array}{l}\text { [81], } \\
{[43],} \\
{[64]}\end{array}$ & & & & [44] & \\
\hline$\zeta_{14}$ & & [44] & & [44] & [44] & $\begin{array}{c}{[4],} \\
{[13],} \\
{[5],} \\
{[77],} \\
{[54],} \\
{[57],} \\
{[47],} \\
{[84],} \\
{[60]}\end{array}$ & & & & & & & & & & & & & & & & $\begin{array}{l}{[5],} \\
{[84]}\end{array}$ & [44] & \\
\hline$\zeta_{15}$ & & & & & & $\begin{array}{l}{[2],} \\
{[43]}\end{array}$ & & & & & [41] & [72] & & & & $\begin{array}{l}\text { [35], } \\
\text { [81] }\end{array}$ & & & $\begin{array}{l}\text { [74], } \\
{[47]}\end{array}$ & & & & & \\
\hline$\zeta_{16}$ & [8] & & & $\begin{array}{l}\text { [4], } \\
{[11]}\end{array}$ & [11] & [14] & [11] & $\begin{array}{l}\text { [41], } \\
{[35]}\end{array}$ & & & $\begin{array}{l}\text { [41], } \\
{[11]}\end{array}$ & & & & & & [11] & [11] & & & & & & \\
\hline$\zeta_{17}$ & & & [95] & & & $\begin{array}{l}{[44],} \\
{[80]}\end{array}$ & & & & & & & & [5] & & & & & & & & & & \\
\hline$\zeta_{18}$ & & & & [4] & & $\begin{array}{l}{[106],} \\
{[44]}\end{array}$ & & & & & & & & & & & & & & & & & & \\
\hline$\zeta_{19}$ & [114] & $\begin{array}{c}{[13],} \\
{[5],} \\
{[55]}\end{array}$ & & $\begin{array}{l}\text { [35], } \\
{[79],} \\
{[13]}\end{array}$ & [13] & & & [5] & $\begin{array}{l}{[72],} \\
{[13]}\end{array}$ & $\begin{array}{l}\text { [73], } \\
{[477]}\end{array}$ & & & $\begin{array}{l}{[35],} \\
{[66],} \\
{[13],} \\
{[47],} \\
{[101],} \\
{[96]}\end{array}$ & & & $\begin{array}{l}\text { [13], } \\
{[39],} \\
{[52]}\end{array}$ & & & & [38] & & $\begin{array}{l}\text { [39], } \\
\text { [97] }\end{array}$ & & [36] \\
\hline$\zeta_{20}$ & & $\begin{array}{l}{[86],} \\
{[59],} \\
{[87]}\end{array}$ & & & [77] & $\begin{array}{l}{[106],} \\
{[85]}\end{array}$ & [14] & & [77] & & & & & & & & & & & & & [83] & & \\
\hline$\zeta_{21}$ & & $\begin{array}{l}\text { [84], } \\
{[104]}\end{array}$ & [104] & & [89] & & [89] & & & & [89] & & [89] & & [84] & & & & & & & & & \\
\hline$\zeta_{22}$ & & $\begin{array}{l}{[58],} \\
{[85],} \\
{[87]}\end{array}$ & & & & $\begin{array}{l}{[57],} \\
{[46],} \\
{[62]}\end{array}$ & [46] & & [93], & [51] & & & & [5] & & & & & & & & & & \\
\hline$\zeta_{23}$ & [44] & $\begin{array}{c}{[5]} \\
{[40]}\end{array}$ & & [3] & & [40] & & & & & [111] & & & & & & & & [80] & & [104] & & & \\
\hline$\zeta_{24}$ & & & & & & & & & & [51] & & & & & & & & & {$[80]$} & & & & & \\
\hline
\end{tabular}


Table 4. Initial reachability matrix of relationships influencing SC elements.

\begin{tabular}{|c|c|c|c|c|c|c|c|c|c|c|c|c|c|c|c|c|c|c|c|c|c|c|c|c|}
\hline & $\zeta_{1}$ & $\zeta_{2}$ & $\zeta_{3}$ & $\zeta_{4}$ & $\zeta_{5}$ & $\zeta_{6}$ & $\zeta_{7}$ & $\zeta_{8}$ & $\zeta_{9}$ & $\zeta_{10}$ & $\zeta_{11}$ & $\zeta_{12}$ & $\zeta_{13}$ & $\zeta_{14}$ & $\zeta_{15}$ & $\zeta_{16}$ & $\zeta_{17}$ & $\zeta_{18}$ & $\zeta_{19}$ & $\zeta_{20}$ & $\zeta_{21}$ & $\zeta_{22}$ & $\zeta_{23}$ & $\zeta_{24}$ \\
\hline$\zeta_{1}$ & 1 & 0 & 0 & 1 & 2 & 2 & 0 & 0 & 0 & 0 & 0 & 0 & 1 & 0 & 0 & 0 & 1 & 0 & 1 & 0 & 0 & 2 & 0 & 0 \\
\hline$\zeta_{2}$ & 3 & 1 & 5 & 0 & 0 & 22 & 3 & 0 & 2 & 1 & 0 & 1 & 0 & 2 & 0 & 1 & 0 & 1 & 4 & 0 & 0 & 3 & 1 & 0 \\
\hline$\zeta_{3}$ & 1 & 4 & 1 & 0 & 0 & 1 & 1 & 0 & 1 & 0 & 0 & 0 & 1 & 1 & 0 & 1 & 1 & 0 & 2 & 0 & 0 & 0 & 1 & 0 \\
\hline$\zeta_{4}$ & 2 & 1 & 0 & 1 & 2 & 10 & 5 & 2 & 1 & 2 & 0 & 2 & 1 & 1 & 0 & 2 & 0 & 0 & 2 & 0 & 0 & 0 & 1 & 0 \\
\hline$\zeta_{6}$ & 0 & 2 & 0 & 0 & 1 & 1 & 3 & 2 & 1 & 1 & 0 & 1 & 1 & 0 & 0 & 1 & 1 & 0 & 3 & 1 & 1 & 0 & 0 & 0 \\
\hline$\zeta_{7}$ & 0 & 2 & 0 & 3 & 1 & 2 & 1 & 1 & 1 & 1 & 0 & 0 & 0 & 1 & 0 & 1 & 2 & 1 & 1 & 0 & 0 & 1 & 0 & 0 \\
\hline$\zeta_{8}$ & 0 & 10 & 0 & 17 & 6 & 7 & 3 & 1 & 2 & 2 & 0 & 0 & 2 & 1 & 1 & 0 & 0 & 0 & 3 & 0 & 1 & 5 & 1 & 0 \\
\hline$\zeta_{9}$ & 2 & 5 & 1 & 1 & 2 & 5 & 1 & 0 & 1 & 1 & 1 & 1 & 1 & 1 & 0 & 1 & 1 & 1 & 4 & 0 & 0 & 2 & 0 & 0 \\
\hline$\zeta_{11}$ & 0 & 0 & 0 & 1 & 0 & 0 & 0 & 2 & 0 & 1 & 1 & 0 & 0 & 0 & 0 & 0 & 0 & 0 & 0 & 0 & 0 & 0 & 2 & 0 \\
\hline$\zeta_{12}$ & 0 & 0 & 0 & 1 & 1 & 0 & 0 & 0 & 0 & 0 & 0 & 1 & 0 & 0 & 1 & 0 & 0 & 0 & 0 & 0 & 0 & 0 & 0 & 0 \\
\hline$\zeta_{13}$ & 0 & 1 & 1 & 0 & 1 & 1 & 1 & 0 & 0 & 1 & 1 & 0 & 1 & 0 & 0 & 0 & 1 & 0 & 3 & 0 & 0 & 0 & 1 & 0 \\
\hline$\zeta_{14}$ & 0 & 1 & 0 & 1 & 1 & 9 & 0 & 0 & 0 & 0 & 0 & 0 & 0 & 1 & 0 & 0 & 0 & 0 & 0 & 0 & 0 & 2 & 1 & 0 \\
\hline$\zeta_{15}$ & 0 & 0 & 0 & 0 & 0 & 2 & 0 & 0 & 0 & 0 & 1 & 1 & 0 & 0 & 1 & 2 & 0 & 0 & 2 & 0 & 0 & 0 & 0 & 0 \\
\hline$\zeta_{16}$ & 1 & 0 & 0 & 2 & 1 & 1 & 1 & 2 & 0 & 0 & 2 & 0 & 0 & 0 & 0 & 1 & 1 & 1 & 0 & 0 & 0 & 0 & 0 & 0 \\
\hline$\zeta_{17}$ & 0 & 0 & 1 & 0 & 0 & 2 & 0 & 0 & 0 & 0 & 0 & 0 & 0 & 1 & 0 & 0 & 1 & 0 & 0 & 0 & 0 & 0 & 0 & 0 \\
\hline$\zeta_{18}$ & 0 & 0 & 0 & 1 & 0 & 2 & 0 & 0 & 0 & 0 & 0 & 0 & 0 & 0 & 0 & 0 & 0 & 1 & 0 & 0 & 0 & 0 & 0 & 0 \\
\hline$\zeta_{19}$ & 1 & 3 & 0 & 3 & 1 & 0 & 0 & 1 & 2 & 2 & 0 & 0 & 6 & 0 & 0 & 3 & 0 & 0 & 1 & 1 & 0 & 2 & 0 & 1 \\
\hline$\zeta_{20}$ & 0 & 3 & 0 & 0 & 1 & 2 & 1 & 0 & 1 & 0 & 0 & 0 & 0 & 0 & 0 & 0 & 0 & 0 & 0 & 1 & 0 & 1 & 0 & 0 \\
\hline$\zeta_{22}$ & 0 & 3 & 0 & 0 & 0 & 3 & 1 & 0 & 2 & 1 & 0 & 0 & 0 & 1 & 0 & 0 & 0 & 0 & 0 & 0 & 0 & 1 & 0 & 0 \\
\hline$\zeta_{23}$ & 1 & 2 & 0 & 1 & 0 & 1 & 0 & 0 & 0 & 0 & 1 & 0 & 0 & 0 & 0 & 0 & 0 & 0 & 1 & 0 & 1 & 0 & 1 & 0 \\
\hline$\zeta_{24}$ & 0 & 0 & 0 & 0 & 0 & 0 & 0 & 0 & 0 & 1 & 0 & 0 & 0 & 0 & 0 & 0 & 0 & 0 & 1 & 0 & 0 & 0 & 0 & 1 \\
\hline
\end{tabular}


Table 5. Final reachability matrix ( $\mathrm{T}=$ transitive link).

\begin{tabular}{|c|c|c|c|c|c|c|c|c|c|c|c|c|c|c|c|c|c|c|c|c|c|c|c|c|c|}
\hline & $\zeta_{1}$ & $\zeta_{2}$ & $\zeta_{3}$ & $\zeta_{4}$ & $\zeta_{5}$ & $\zeta_{6}$ & $\zeta_{7}$ & $\zeta_{8}$ & $\zeta_{9}$ & $\zeta_{10}$ & $\zeta_{11}$ & $\zeta_{12}$ & $\zeta_{13}$ & $\zeta_{14}$ & $\zeta_{15}$ & $\zeta_{16}$ & $\zeta_{17}$ & $\zeta_{18}$ & $\zeta_{19}$ & $\zeta_{20}$ & $\zeta_{21}$ & $\zeta_{22}$ & $\zeta_{23}$ & $\zeta_{24}$ & DRV \\
\hline$\zeta_{1}$ & 1 & $\mathrm{~T}$ & $\mathrm{~T}$ & 1 & 2 & 2 & $\mathrm{~T}$ & $\mathrm{~T}$ & $\mathrm{~T}$ & $\mathrm{~T}$ & $\mathrm{~T}$ & $\mathrm{~T}$ & 1 & $\mathrm{~T}$ & $\mathrm{~T}$ & $\mathrm{~T}$ & 1 & $\mathrm{~T}$ & 1 & $\mathrm{~T}$ & $\mathrm{~T}$ & 2 & $\mathrm{~T}$ & $\mathrm{~T}$ & 24 \\
\hline$\zeta_{2}$ & 3 & 1 & 5 & $\mathrm{~T}$ & $\mathrm{~T}$ & 22 & 3 & $\mathrm{~T}$ & 2 & 1 & $\mathrm{~T}$ & 1 & $\mathrm{~T}$ & 2 & $\mathrm{~T}$ & 1 & $\mathrm{~T}$ & 1 & 4 & $\mathrm{~T}$ & $\mathrm{~T}$ & 3 & 1 & $\mathrm{~T}$ & 23 \\
\hline$\zeta_{3}$ & 1 & 4 & 1 & $\mathrm{~T}$ & $\mathrm{~T}$ & 1 & 1 & $\mathrm{~T}$ & 1 & $\mathrm{~T}$ & $\mathrm{~T}$ & $\mathrm{~T}$ & 1 & 1 & 0 & 1 & 1 & $\mathrm{~T}$ & 2 & $\mathrm{~T}$ & $\mathrm{~T}$ & $\mathrm{~T}$ & 1 & $\mathrm{~T}$ & 23 \\
\hline$\zeta_{4}$ & 2 & 1 & $\mathrm{~T}$ & 1 & 2 & 10 & 5 & 2 & 1 & 2 & $\mathrm{~T}$ & 2 & 1 & 1 & $\mathrm{~T}$ & 2 & $\mathrm{~T}$ & $\mathrm{~T}$ & 2 & $\mathrm{~T}$ & $\mathrm{~T}$ & $\mathrm{~T}$ & 1 & $\mathrm{~T}$ & 24 \\
\hline$\zeta_{5}$ & 1 & 12 & $\mathrm{~T}$ & 4 & 1 & 2 & 2 & 2 & 2 & 2 & 3 & $\mathrm{~T}$ & 1 & 3 & $\mathrm{~T}$ & 2 & 1 & 1 & 7 & 1 & 1 & 3 & $\mathrm{~T}$ & $\mathrm{~T}$ & 24 \\
\hline$\zeta_{6}$ & $\mathrm{~T}$ & 2 & $\mathrm{~T}$ & 0 & 1 & 1 & 3 & 2 & 1 & 1 & $\mathrm{~T}$ & 1 & 1 & $\mathrm{~T}$ & $\mathrm{~T}$ & 1 & 1 & $\mathrm{~T}$ & 3 & 1 & 1 & $\mathrm{~T}$ & $\mathrm{~T}$ & $\mathrm{~T}$ & 23 \\
\hline$\zeta_{7}$ & $\mathrm{~T}$ & 2 & $\mathrm{~T}$ & 3 & 1 & 2 & 1 & 1 & 1 & 1 & $\mathrm{~T}$ & $\mathrm{~T}$ & $\mathrm{~T}$ & 1 & $\mathrm{~T}$ & 1 & 2 & 1 & 1 & $\mathrm{~T}$ & 0 & 1 & $\mathrm{~T}$ & $\mathrm{~T}$ & 22 \\
\hline$\zeta_{8}$ & $\mathrm{~T}$ & 10 & $\mathrm{~T}$ & 17 & 6 & 7 & 3 & 1 & 2 & 2 & $\mathrm{~T}$ & $\mathrm{~T}$ & 2 & 1 & 1 & $\mathrm{~T}$ & $\mathrm{~T}$ & $\mathrm{~T}$ & 3 & $\mathrm{~T}$ & 1 & 5 & 1 & $\mathrm{~T}$ & 24 \\
\hline$\zeta_{9}$ & 2 & 5 & 1 & 1 & 2 & 5 & 1 & $\mathrm{~T}$ & 1 & 1 & 1 & 1 & 1 & 1 & $\mathrm{~T}$ & 1 & 1 & 1 & 4 & $\mathrm{~T}$ & $\mathrm{~T}$ & 2 & $\mathrm{~T}$ & $\mathrm{~T}$ & 24 \\
\hline$\zeta_{10}$ & 1 & 2 & $\mathrm{~T}$ & 1 & 6 & 2 & $\mathrm{~T}$ & 1 & 1 & 1 & 3 & $\mathrm{~T}$ & 5 & $\mathrm{~T}$ & $\mathrm{~T}$ & $\mathrm{~T}$ & $\mathrm{~T}$ & $\mathrm{~T}$ & $\mathrm{~T}$ & $\mathrm{~T}$ & $\mathrm{~T}$ & 2 & $\mathrm{~T}$ & 0 & 24 \\
\hline$\zeta_{11}$ & $\mathrm{~T}$ & $\mathrm{~T}$ & 0 & 1 & $\mathrm{~T}$ & $\mathrm{~T}$ & $\mathrm{~T}$ & 2 & $\mathrm{~T}$ & 1 & 1 & $\mathrm{~T}$ & $\mathrm{~T}$ & $\mathrm{~T}$ & $\mathrm{~T}$ & $\mathrm{~T}$ & 0 & 0 & $\mathrm{~T}$ & 0 & $\mathrm{~T}$ & $\mathrm{~T}$ & 2 & 0 & 19 \\
\hline$\zeta_{12}$ & $\mathrm{~T}$ & $\mathrm{~T}$ & 0 & 1 & 1 & $\mathrm{~T}$ & $\mathrm{~T}$ & $\mathrm{~T}$ & $\mathrm{~T}$ & $\mathrm{~T}$ & $\mathrm{~T}$ & 1 & $\mathrm{~T}$ & $\mathrm{~T}$ & 1 & $\mathrm{~T}$ & $\mathrm{~T}$ & $\mathrm{~T}$ & $\mathrm{~T}$ & $\mathrm{~T}$ & $\mathrm{~T}$ & $\mathrm{~T}$ & $\mathrm{~T}$ & 0 & 23 \\
\hline$\zeta_{13}$ & $\mathrm{~T}$ & 1 & 1 & $\mathrm{~T}$ & 1 & 1 & 1 & $\mathrm{~T}$ & $\mathrm{~T}$ & 1 & 1 & $\mathrm{~T}$ & 1 & $\mathrm{~T}$ & 0 & $\mathrm{~T}$ & 1 & $\mathrm{~T}$ & 3 & $\mathrm{~T}$ & $\mathrm{~T}$ & $\mathrm{~T}$ & 1 & $\mathrm{~T}$ & 23 \\
\hline$\zeta_{14}$ & $\mathrm{~T}$ & 1 & $\mathrm{~T}$ & 1 & 1 & 9 & $\mathrm{~T}$ & $\mathrm{~T}$ & $\mathrm{~T}$ & $\mathrm{~T}$ & $\mathrm{~T}$ & $\mathrm{~T}$ & $\mathrm{~T}$ & 1 & 0 & $\mathrm{~T}$ & $\mathrm{~T}$ & $\mathrm{~T}$ & $\mathrm{~T}$ & $\mathrm{~T}$ & $\mathrm{~T}$ & 2 & 1 & 0 & 23 \\
\hline$\zeta_{15}$ & $\mathrm{~T}$ & $\mathrm{~T}$ & 0 & $\mathrm{~T}$ & $\mathrm{~T}$ & 2 & $\mathrm{~T}$ & $\mathrm{~T}$ & $\mathrm{~T}$ & $\mathrm{~T}$ & 1 & 1 & $\mathrm{~T}$ & 0 & 1 & 2 & $\mathrm{~T}$ & 0 & 2 & $\mathrm{~T}$ & $\mathrm{~T}$ & $\mathrm{~T}$ & $\mathrm{~T}$ & $\mathrm{~T}$ & 20 \\
\hline$\zeta_{16}$ & 1 & $\mathrm{~T}$ & $\mathrm{~T}$ & 2 & 1 & 1 & 1 & 2 & $\mathrm{~T}$ & $\mathrm{~T}$ & 2 & $\mathrm{~T}$ & $\mathrm{~T}$ & $\mathrm{~T}$ & $\mathrm{~T}$ & 1 & 1 & 1 & $\mathrm{~T}$ & $\mathrm{~T}$ & $\mathrm{~T}$ & $\mathrm{~T}$ & $\mathrm{~T}$ & 0 & 24 \\
\hline$\zeta_{17}$ & $\mathrm{~T}$ & $\mathrm{~T}$ & 1 & $\mathrm{~T}$ & $\mathrm{~T}$ & 2 & $\mathrm{~T}$ & $\mathrm{~T}$ & $\mathrm{~T}$ & $\mathrm{~T}$ & 0 & $\mathrm{~T}$ & $\mathrm{~T}$ & 1 & 0 & $\mathrm{~T}$ & 1 & 0 & $\mathrm{~T}$ & $\mathrm{~T}$ & $\mathrm{~T}$ & $\mathrm{~T}$ & $\mathrm{~T}$ & 0 & 20 \\
\hline$\zeta_{18}$ & $\mathrm{~T}$ & $\mathrm{~T}$ & 0 & 1 & $\mathrm{~T}$ & 2 & $\mathrm{~T}$ & $\mathrm{~T}$ & $\mathrm{~T}$ & $\mathrm{~T}$ & 0 & $\mathrm{~T}$ & $\mathrm{~T}$ & $\mathrm{~T}$ & 0 & $\mathrm{~T}$ & $\mathrm{~T}$ & 1 & $\mathrm{~T}$ & $\mathrm{~T}$ & $\mathrm{~T}$ & 0 & $\mathrm{~T}$ & 0 & 19 \\
\hline$\zeta_{19}$ & 1 & 3 & $\mathrm{~T}$ & 3 & 1 & $\mathrm{~T}$ & $\mathrm{~T}$ & 1 & 2 & 2 & $\mathrm{~T}$ & $\mathrm{~T}$ & 6 & $\mathrm{~T}$ & $\mathrm{~T}$ & 3 & $\mathrm{~T}$ & $\mathrm{~T}$ & 1 & 1 & $\mathrm{~T}$ & 2 & $\mathrm{~T}$ & 1 & 24 \\
\hline$\zeta_{20}$ & $\mathrm{~T}$ & 3 & $\mathrm{~T}$ & $\mathrm{~T}$ & 1 & 2 & 1 & $\mathrm{~T}$ & 1 & $\mathrm{~T}$ & $\mathrm{~T}$ & $\mathrm{~T}$ & $\mathrm{~T}$ & $\mathrm{~T}$ & 0 & $\mathrm{~T}$ & $\mathrm{~T}$ & $\mathrm{~T}$ & $\mathrm{~T}$ & 1 & $\mathrm{~T}$ & 1 & $\mathrm{~T}$ & 0 & 23 \\
\hline$\zeta_{21}$ & $\mathrm{~T}$ & 2 & 1 & $\mathrm{~T}$ & 1 & $\mathrm{~T}$ & 1 & $\mathrm{~T}$ & $\mathrm{~T}$ & $\mathrm{~T}$ & 1 & $\mathrm{~T}$ & 1 & $\mathrm{~T}$ & 1 & $\mathrm{~T}$ & $\mathrm{~T}$ & $\mathrm{~T}$ & $\mathrm{~T}$ & $\mathrm{~T}$ & 1 & $\mathrm{~T}$ & $\mathrm{~T}$ & 0 & 24 \\
\hline$\zeta_{22}$ & $\mathrm{~T}$ & 3 & $\mathrm{~T}$ & $\mathrm{~T}$ & $\mathrm{~T}$ & 3 & 1 & $\mathrm{~T}$ & 2 & 1 & $\mathrm{~T}$ & $\mathrm{~T}$ & $\mathrm{~T}$ & 1 & 0 & $\mathrm{~T}$ & $\mathrm{~T}$ & $\mathrm{~T}$ & $\mathrm{~T}$ & $\mathrm{~T}$ & $\mathrm{~T}$ & 1 & $\mathrm{~T}$ & 0 & 22 \\
\hline$\zeta_{23}$ & 1 & 2 & 0 & 1 & $\mathrm{~T}$ & 1 & $\mathrm{~T}$ & $\mathrm{~T}$ & $\mathrm{~T}$ & $\mathrm{~T}$ & 1 & 0 & $\mathrm{~T}$ & $\mathrm{~T}$ & 0 & $\mathrm{~T}$ & 0 & 0 & 1 & $\mathrm{~T}$ & 1 & $\mathrm{~T}$ & 1 & $\mathrm{~T}$ & 18 \\
\hline$\zeta_{24}$ & $\mathrm{~T}$ & $\mathrm{~T}$ & 0 & $\mathrm{~T}$ & $\mathrm{~T}$ & $\mathrm{~T}$ & 0 & $\mathrm{~T}$ & $\mathrm{~T}$ & 1 & $\mathrm{~T}$ & 0 & $\mathrm{~T}$ & 0 & 0 & $\mathrm{~T}$ & 0 & 0 & 1 & $\mathrm{~T}$ & 0 & $\mathrm{~T}$ & 0 & 1 & 16 \\
\hline DEP. & 24 & 24 & 18 & 23 & 24 & 24 & 24 & 24 & 24 & 24 & 21 & 22 & 24 & 23 & 15 & 24 & 21 & 19 & 24 & 23 & 22 & 23 & 23 & 16 & \\
\hline
\end{tabular}


Table 6. Intersection of reachability and antecedent sets and presentation of the Level 1 group.

\begin{tabular}{|c|c|c|c|c|}
\hline Var. & Reachability Set & Antecedent Set & Intersection & Level \\
\hline \multicolumn{5}{|c|}{ Iteration 1} \\
\hline$\zeta_{1}$ & $1234 \ldots 12131415161718192021222324$ & $1234 \ldots 12131415161718192021222324$ & $1234 \ldots 12131415161718192021222324$ & I \\
\hline$\zeta_{2}$ & $1234 \ldots 121314151617181920212223$ & $1234 \ldots 12131415161718192021222324$ & $1234 \ldots 121314151617181920212223$ & I \\
\hline$\zeta_{5}$ & $1234 \ldots 12131415161718192021222324$ & $1234 \ldots 12131415161718192021222324$ & $1234 \ldots 12131415161718192021222324$ & $\mathrm{I}$ \\
\hline$\zeta_{6}$ & $1235 \ldots 12131415161718192021222324$ & $1234 \ldots 12131415161718192021222324$ & $1235 \ldots 12131415161718192021222324$ & I \\
\hline$\zeta_{7}$ & $1234 \ldots 1213141516171819202223$ & $1234 \ldots 12131415161718192021222324$ & $1234 \ldots 1213141516171819202223$ & I \\
\hline$\zeta_{8}$ & $1234 \ldots 12131415161718192021222324$ & $1234 \ldots 12131415161718192021222324$ & $1234 \ldots 12131415161718192021222324$ & $\mathrm{I}$ \\
\hline$\zeta_{9}$ & $1234 \ldots 12131415161718192021222324$ & $1234 \ldots 12131415161718192021222324$ & $1234 \ldots 12131415161718192021222324$ & I \\
\hline$\zeta_{10}$ & $1234 \ldots 12131415161718192021222324$ & $1234 \ldots 12131415161718192021222324$ & $1234 \ldots 12131415161718192021222324$ & $\mathrm{I}$ \\
\hline$\zeta_{11}$ & $124 \ldots 121314151619212223$ & $1234 \ldots 12131415161920212223$ & $124 \ldots 121314151619212223$ & I \\
\hline$\zeta_{13}$ & $1234 \ldots 121314161718192021222324$ & $1234 \ldots 12131415161718192021222324$ & $1234 \ldots 121314161718192021222324$ & I \\
\hline$\zeta_{14}$ & $1234 \ldots 121314161718192021222324$ & $1234 \ldots 121314161718192021222324$ & $1234 \ldots 121314161718192021222324$ & I \\
\hline$\zeta_{19}$ & $1234 \ldots 12131415161718192021222324$ & $1234 \ldots 12131415161718192021222324$ & $1234 \ldots 12131415161718192021222324$ & I \\
\hline$\zeta_{23}$ & $124 \ldots 111314161920212223$ & $1234 \ldots 121314151617181920212223$ & $124 \ldots 111314161920212223$ & I \\
\hline \multicolumn{5}{|c|}{ Iteration 2} \\
\hline$\zeta_{4}$ & 34121516171820212224 & 34121516171820212224 & 34121516171820212224 & II \\
\hline$\zeta_{16}$ & 34121516171820212224 & 34121516171820212224 & 34121516171820212224 & II \\
\hline$\zeta_{17}$ & 34121617202122 & 341215161718202122 & 34121617202122 & II \\
\hline$\zeta_{18}$ & 121821 & 312182122 & 121821 & III \\
\hline \multicolumn{5}{|c|}{ Iteration 4} \\
\hline$\zeta_{22}$ & 3122122 & 31215212224 & 3122122 & IV \\
\hline \multicolumn{5}{|c|}{ Iteration 5} \\
\hline$\zeta_{15}$ & 121521 & 121521 & 121521 & $\mathrm{~V}$ \\
\hline$\zeta_{24}$ & 24 & 3122124 & 24 & V \\
\hline \multicolumn{5}{|c|}{ Iteration 6} \\
\hline$\zeta_{12}$ & 1221 & 31221 & 1221 & VI \\
\hline$\zeta_{21}$ & 31221 & 31221 & 31221 & VI \\
\hline \multicolumn{5}{|c|}{ Iteration 7} \\
\hline$\zeta_{3}$ & 321 & 321 & 321 & VII \\
\hline
\end{tabular}


After iterating the SC elements at different levels, digraphs are used to depict the hierarchical relationships of influence based on the final reachability matrix. The total of seven levels of SC capabilities are then identified. The first level consists of the considered SC Network Design $\left(\zeta_{1}\right)$; Flexibility $\left(\zeta_{2}\right)$; Collaboration $\left(\zeta_{5}\right)$; Agility and Responsiveness $\left(\zeta_{6}\right)$; Alertness, Awareness, and Anticipation $\left(\zeta_{7}\right)$; IT and Information Sharing $\left(\zeta_{8}\right)$; Robustness $\left(\zeta_{9}\right)$; SCRM Culture $\left(\zeta_{10}\right)$; Security $\left(\zeta_{11}\right)$; SC Continuity and Contingency Planning $\left(\zeta_{13}\right)$; Velocity $\left(\zeta_{14}\right)$; Risk Control $\left(\zeta_{19}\right)$; and, Trust $\left(\zeta_{23}\right)$. Level 2 consists of Visibility $\left(\zeta_{4}\right)$, Integration $\left(\zeta_{16}\right)$, Adaptability $\left(\zeta_{17}\right)$, and Alignment $\left(\zeta_{20}\right)$. Levels 3 and 4 consist of Market Position $\left(\zeta_{18}\right)$ and Efficiency $\left(\zeta_{22}\right)$, respectively. Level 5 consists of Coordination $\left(\zeta_{15}\right)$ and Sustainability $\left(\zeta_{24}\right)$. Level 6 consists of Knowledge Management $\left(\zeta_{12}\right)$ and PPP $\left(\zeta_{21}\right)$. Finally, Level 7 consists of Redundancy $\left(\zeta_{3}\right)$.

\section{Results and Discussion}

Our study reveals the interactions and the resulting interdependent structural relationships among SCRES capabilities and elements. Our proposed evidence-based approach contributes to the stream of literature on SCRES by lending (i) support and updates to previous research that examined the core SCRES capabilities, and (ii) complementary classifications of influential powers of SCRES capabilities and elements.

\subsection{Evidence-based ISM Driven Hierarchical Level of SCRES Capabilities}

Three types of investigations of SC resilience are observed: Qualitative, quantitative, and explorative. Based on the qualitative approach, such as the case study or systematic literature review, Machado et al. [115] identified 11 mitigation capabilities and found that companies develop different levels of proactivity and capabilities needed for counterfeiting resilience. Similarly, Stone and Rahimifard [18] identified 23 SC capabilities and highlighted that resilience must concern the ability to not only maintain core function, but also adapt to changing conditions. Ali et al. [6] and Hohenstein et al. [13] both performed explorative studies based on SLR and discovered 13 and six SCRES elements, respectively. Both studies mainly contributed to conceptual identification of resilience based on an integrated view of the resilience concept and SCRES strategies. These studies commonly examined the literature underpinning the review question (i.e., 'what are the essential elements and managerial practices needed to support SCRES capabilities?'), but does not further provide a strategic managerial guideline. Similarly, from the quantitative perspective, Kim et al. [8] operationalized SCRES as relative number of disruptions in a network and noted the importance of differentiating node and arc-level disruption to a network disruption. While the implication of different structural relationships among network entities are emphasized, strategic SC resilience management planning is not discussed. This study provides both a qualitative and quantitative approach to identify interrelationship driven hierarchical levels of SCRES capabilities and extract key performance measures based on SLR and ISM analyses. Moreover, the practitioners can develop a strategic SCRES management guideline based on influential characteristics of each SCRES capabilities.

The evidence-based ISM model of the SC capabilities for SCRES indicates the interrelationship between different SC capabilities and illustrates its levels determined based on systematic iterations. The final digraph (Figure 2) can assist practitioners in resilience management planning for SC sustainability.

The finding implies that 'Redundancy' (Level VII) will help in achieving 'Knowledge Management' and 'PPP' (Level VI), which will then positively drive 'Collaboration' and 'Sustainability' (Level V), ultimately achieving 'Efficiency' (Level IV). It will also improve SC capabilities associated with Level III and Level II. Finally, all these SC capabilities will lead to a positive outcome of Level I SC capabilities, which can be deemed as the final performance measure of SCRES. At intermediate levels of the digraph, Level II, which has a direct contribution to Level I, is composed of visibility, integration, adaptability, and alignment. This is aligned with Scholten and Schilder's [84] statement that collaboration is essential 
for building resilience. The authors found that firms with higher alignment and integration with increased visibility will offer higher resilience performance compared to a newly engaging supplier.

The primary representative elements or performance measures for SCRES are considered to be 'SC Network Design', 'Flexibility', 'Collaboration', 'Agility and Responsiveness', 'Alertness, Awareness, and Anticipation', 'IT and Information Sharing', 'Robustness', 'SC Risk Management culture', 'Security', 'SC Continuity', 'Velocity', 'Risk Control', and 'Trust'. Similar results are found in a resilience context. A notable difference between Ali et al.'s [6] most recent finding of SCRES elements based on the SLR investigation between 2000 and 2015 is the inclusion of 'SC Network Design'. The structure of the SC network plays a vital role in solving network complexity and mitigating risky situations $[14,70,101]$. Successful SC network reconfiguration enhances visibility and helps identify disruptive events in a timely manner by combining strong and weak ties among the SC network [44,72]. The imperativeness of network design is highlighted for its effectiveness on all other network characteristics as well [51]. Specifically, network clustering, complexity, and connectivity determine supply chain robustness and resilience [39].

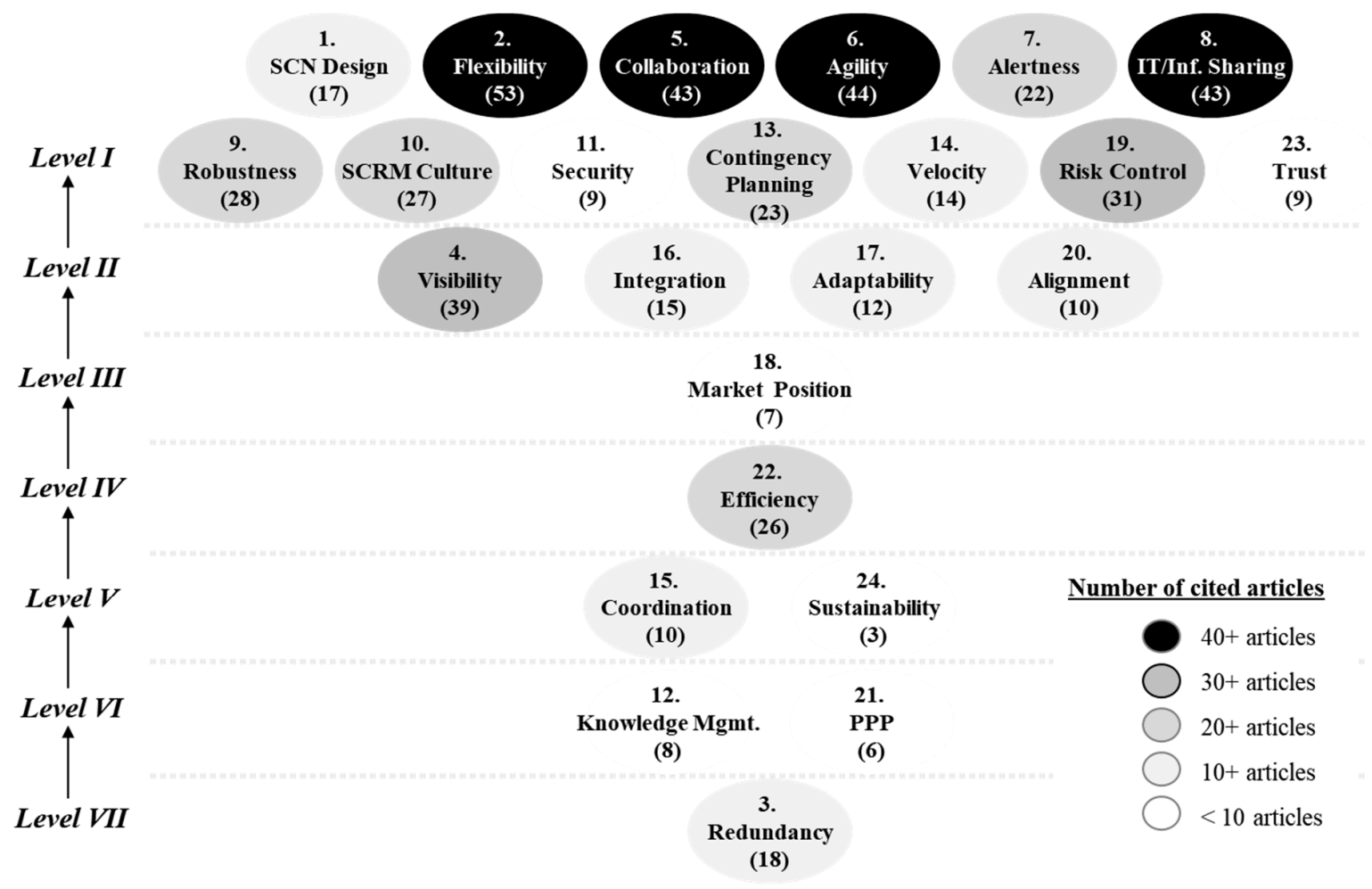

Figure 2. Digraph of SC resilience capability relationships.

\subsection{MICMAC-based Four Types of Classification of SC Capabilities}

As a complementary analysis to the ISM-based level partitioning analysis, a MICMAC (Méthode de hiérarchisation des éléments d'un système) analysis is conducted to classify the elements variables in four types of variables (autonomous, dependent, linkage, independent) based on their driving and dependence powers [116] (Figure 3). The advantage of MICMAC is the identification of the influence level of each capability on the others. The driving power of a variable is determined by the total number of variables it affects, whereas the dependence power is determined by the total number of variables that affect the variable under investigation. The result can be utilized to assist practitioners in prioritizing the improvement planning of capabilities. 


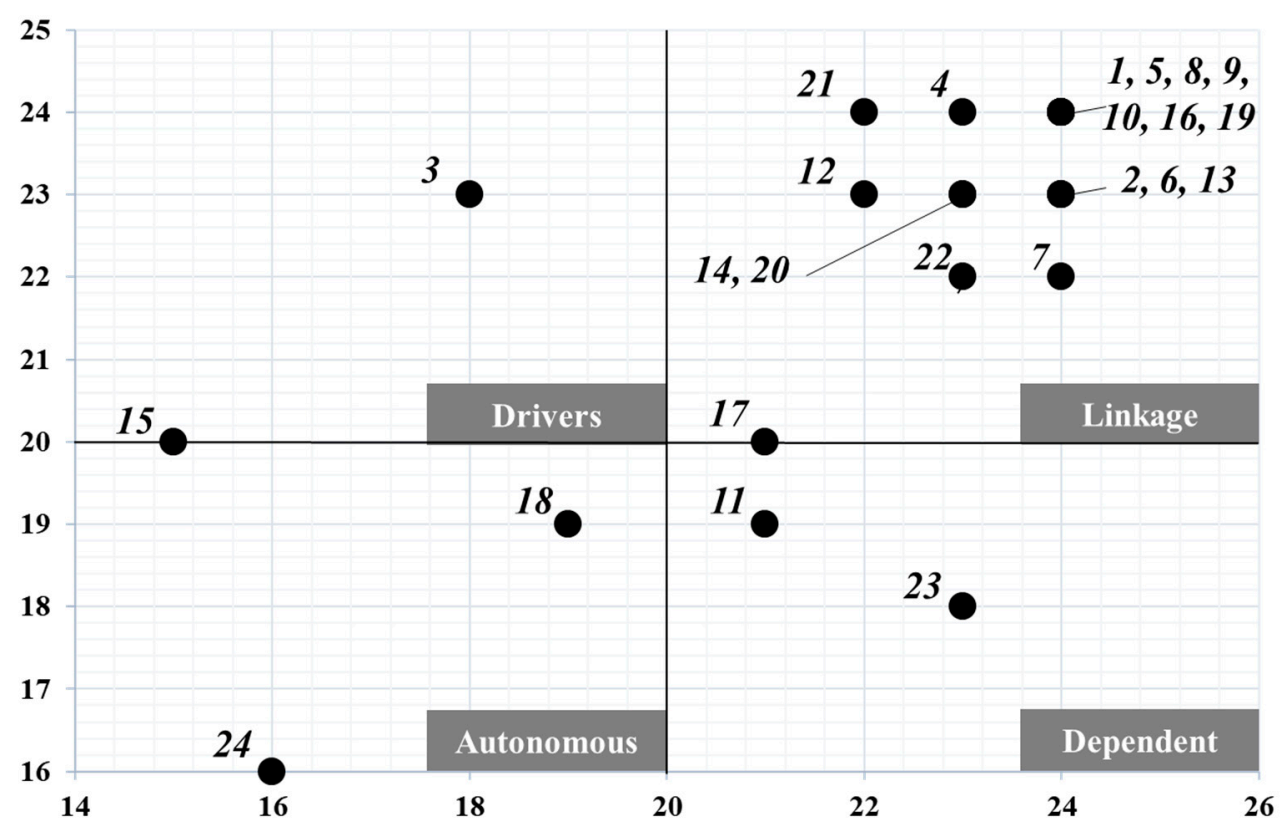

Figure 3. MICMAC analysis for influential SC capabilities in a resilience context (the x-axis and the $\mathrm{y}$-axis represent the levels of dependence and driving power, respectively).

Overall, 'Redundancy' as the lowest-tier of the ISM model (Level VII) is confirmed as an important driving force of SCRES. 'Independent' capability (upper-left quadrant), with a high driving and low dependence power, is redundancy $\left(\zeta_{3}\right)$. This independent capability significantly influences other capabilities, thereby ultimately shaping the overall outcome of the system. Redundancy, through additional inventory and capacity building, enables the response to disruptive events through multiple suppliers and slack resources in production and/or transportation $[13,117]$. Redundancy has been consistently pointed as an antecedent of a wide range of-if not of all-SC elements, such as flexibility, responsiveness, efficiency, robustness, and contingency planning [44,64]. Although redundancy is regarded as one of many attributes that route to flexibility-based resilience [5], it is also highlighted for its uniqueness in ensuring key performances, such as SC continuity, when the entire supply market is exposed to risks [64].

'Dependent' capabilities (lower-right quadrant), characterized by low driving and high dependence powers, are security $\left(\zeta_{11}\right)$, adaptability $\left(\zeta_{17}\right)$, and trust $\left(\zeta_{23}\right)$. The effects of these elements are contingent on the effect of the 'independent' and 'linkage' elements. Despite their significant role in SC network resilience, these elements' performances are highly influenced by other elements. A high level of adaptability requires requisite quantities of redundancy, in addition to flexibility and collaboration $[1,5,51]$. These three elements are commonly positioned at the intermediate levels of the ISM model (Level I and II) with a low number of articles referenced (less than 20). Based on the social exchange theory, interconnected firms are advised to work towards commitment and attachment to minimize risks of business uncertainties [118]. While the value of such exchange relationship driven capabilities are highlighted for its resulting positive transaction relationship, these capabilities are frequently referred as indirect mitigators rather than core determinants of supply chain outcomes [119]. This finding implies that these capabilities provide limited impact on SC resilience outcomes and are highly determined by the performances of other capabilities, such as 'driving' and 'linkage' capabilities.

'Autonomous' capabilities (lower-left quadrant) with low driving and dependence levels are the market position $\left(\zeta_{18}\right)$ and sustainability $\left(\zeta_{24}\right)$. Although the market position represents the status of a company or its product [11], sustainability represents the status of a firm's responsibility with respect to ethical and environmental issues [34,56]. Firms' strategic directions of product differentiation, brand reputation, and customer retention planning are standalone SC elements measured and evaluated for the overall resilience performance. This finding indicates that these capabilities are 
relatively disconnected from the other, and must be treated as distinct capabilities for the desired level of resilience. For example, sustainability has been regarded as a broad-level concern, but is continuously undergoing investigation in how it can be incorporated into strategic and operational levels of management decision-making [26]. Aligned with Sheffi and Rice's [1] finding that firms with market power can quickly recover from disruption and benefit from resiliency, requirements for successful SCRES include strategic management decisions, such as the development of supplier selection strategies based on sustainability [47] and the consideration of the market share among SC members [120].

Most importantly, our findings suggest that most factors with high driving and dependence power are key SC capabilities, which are identified in Level I. This finding can be utilized to set up an effective and efficient strategy that supports and prepares the firms in SCRES management. 'Linkage' capabilities (upper-right quadrant) simultaneously reflect high driving and dependence powers. Not only do these elements drive the system, but they are also dependent on other elements. These elements play a mediating role in transferring effects to other elements, including to themselves. Out of the 24 capabilities, 17 pertain to this category: SC network design $\left(\zeta_{1}\right)$, flexibility $\left(\zeta_{2}\right)$, visibility $\left(\zeta_{4}\right)$, collaboration $\left(\zeta_{5}\right)$, agility $\left(\zeta_{6}\right)$, alertness, awareness, and anticipation $\left(\zeta_{7}\right)$, IT and information sharing $\left(\zeta_{8}\right)$, robustness $\left(\zeta_{9}\right)$, SCRM culture $\left(\zeta_{10}\right)$, knowledge management $\left(\zeta_{12}\right)$, contingency planning $\left(\zeta_{13}\right)$, velocity $\left(\zeta_{14}\right)$, integration $\left(\zeta_{16}\right)$, risk control $\left(\zeta_{19}\right)$, alignment $\left(\zeta_{20}\right)$, PPP $\left(\zeta_{21}\right)$, and efficiency $\left(\zeta_{22}\right)$. The effective performance of these elements will not only boost SC network resilience, but it will also positively impact the other system elements as well. For example, improving PPP, which is composed of public and private entities attempting to create global sustainable value [121], will directly impact the overall SC network resilience performance, and also increase levels of visibility and velocity through the joint relationship effort [84]. Similar with recent findings [13,18,84,115], flexibility, collaboration, agility, alertness, and IT and information sharing have been predominantly noted as key SCRES capabilities with exceptionally higher dependency powers. Interestingly, while SC network design has not been frequently included as a core SCRES capability, our finding indicates the necessity of inclusion of SC network design for its linkage role. The imperativeness of network design and its characteristics (such as network clustering, complexity, connectivity) has gained attention for leading to a higher level of SC resilience [39,72]. An appropriate structure of the SC network may effectively bolster supply network resilience by either enhancing visibility or building flexibility and agility towards disruptive events.

\section{Conclusions and Future Studies}

Theory-based resilience management is increasingly becoming a major objective for both local and globally connected suppliers and buyers to respond to disruptive events [122]. The proactive attainment of resilience not only ensures absorptivity of and adaptability to sudden changes, but also offers a competitive advantage through a distinct level of the recovery and regrowth process $[123,124]$. SCRES practices improve the sustainability of traditional SC network systems by considering network relationships, environmental issues, and obtainable competitive advantages. In line with previous research, we identified and updated 13 key SC capabilities out of 24 as performance measures of SC network resilience. Furthermore, our findings categorised each capability into one of four different types of influential power.

From a theoretical perspective, researchers can benefit from elaboration of the understanding of key performance measures for SC network resilience. Based on the repository of literature, both researchers and practitioners can collaboratively and structurally investigate the interdependent relationships among resilience elements through the application of the proposed evidence-based ISM methodology. Both theoretical and empirical findings-driven literature leads to hypothetical models that can be efficiently constructed, especially with more than 10 capabilities or elements as potential determinants of the performance under investigation. The existing diversity in the resilience context—such as in ecological system sciences, psychology, SCs, and the community-has resulted in 
a fragmented, inconsistent understanding of definitions, elements, and strategies [18]. The proposed analysis is recommended for systematically identifying key principles and building a foundation for any highly fragmented research context for better elaboration.

From a managerial perspective, the implications are mainly twofold: Increasing the awareness of resilience and its interconnected SC elements and visualizing their influential characterization: (i) The evidence-based ISM analysis of existing SCRES studies reveals the interdependent structural relationships among SCRES elements (Figure 2). These findings first provide useful information for practitioners to proactively plan for the varying cases of operation management situations based on 24 SC elements. The firm may wish to focus only on achieving high levels of capabilities closely related to resilience performance (Level I), or of capabilities directly and indirectly enabling resilience management overall (Levels I through VI). (ii) MICMAC-based classification of capabilities according to their level of influence (Figure 3) offers a means to develop a strategic, yet efficiently resilient, SC network management portfolio. Although improving multiple capabilities simultaneously may be an optimal solution, it may require significant budget and resource investments. For example, Chaing et al.'s [69] discovery of the flexibility-agility relationship could simultaneously improve both capabilities, such as the flexibility in the supply design process, and efforts in customer/demand responsiveness and joint planning. However, Gligor et al. [125] recommended primary improvement of 'agility' based on a classification from a financial management perspective.

Future research may further validate our findings and provide other valuable contributions. First, future research could explore the structural relationships among SC elements based on different phases of a disruption (pre-, during-, and post-disruption). Second, academic researchers as well as practitioners could benefit from a thorough understanding of system dynamics and performance behaviours in these varying situations. Lastly, this study encourages future researchers to empirically confirm the findings in various industries and contexts.

Author Contributions: All authors conceived, developed, analyzed the research model; contributed to the discussion and design of the framework; and wrote the manuscript. All authors read and approved the final manuscript.

Acknowledgments: The authors thank the Institute of Management Research at Seoul National University for supporting this research.

Conflicts of Interest: The authors declare no conflicts of interest.

\section{References}

1. Sheffi, Y.; Rice, J.B., Jr. A supply chain view of the resilient enterprise. MIT Sloan Manag. Rev. 2005, 47, 41-48.

2. Ponomarov, S.Y.; Holcomb, M.C. Understanding the concept of supply chain resilience. Int. J. Logist. Manag. 2009, 20, 124-143. [CrossRef]

3. Pettit, T.J.; Fiksel, J.; Croxton, K.L. Ensuring supply chain resilience: Development of a conceptual framework. J. Bus. Logist. 2010, 31, 1-21. [CrossRef]

4. Christopher, M.; Peck, H. Building the resilient supply chain. Int. J. Logist. Manag. 2004, 15, 1-14. [CrossRef]

5. Jüttner, U.; Maklan, S. Supply chain resilience in the global financial crisis: An empirical study. Supply Chain Manag. An Int. J. 2011, 16, 246-259. [CrossRef]

6. Ali, A.; Mahfouz, A.; Arisha, A. Analysing supply chain resilience: integrating the constructs in a concept mapping framework via a systematic literature review. Supply Chain Manag. An Int. J. 2017, 22, 16-39. [CrossRef]

7. Ponte, B.; Fernández, I.; García, N.; Pino, R.; de la Fuente, D. Supply Chain Management: The Systemic Challenge. In Advances in Management Engineering; Springer: Gewerbestrasse, Switzerland, 2017; pp. $109-119$. ISBN 978-3-319-55888-2.

8. Kim, Y.; Chen, Y.S.; Linderman, K. Supply network disruption and resilience: A network structural perspective. J. Oper. Manag. 2015, 33-34, 43-59. [CrossRef]

9. Dominguez, R.; Cannella, S.; Framinan, J.M. On bullwhip-limiting strategies in divergent supply chain networks. Comput. Ind. Eng. 2014, 73, 85-95. [CrossRef] 
10. Fan, Y.; Stevenson, M. A review of supply chain risk management: definition, theory, and research agenda. Int. J. Phys. Distrib. Logist. Manag. 2018, 48, 205-230. [CrossRef]

11. Pettit, T.J.; Croxton, K.L.; Fiksel, J. Ensuring supply chain resilience: Development and implementation of an assessment tool. J. Bus. Logist. 2013, 34, 46-76. [CrossRef]

12. Bhamra, R.; Dani, S.; Burnard, K. Resilience: the concept, a literature review and future directions. Int. J. Prod. Res. 2011, 49, 5375-5393. [CrossRef]

13. Hohenstein, N.-O.; Feisel, E.; Hartmann, E.; Giunipero, L. Research on the phenomenon of supply chain resilience. Int. J. Phys. Distrib. Logist. Manag. 2015, 45, 90-117. [CrossRef]

14. Roberta Pereira, C.; Christopher, M.; Lago Da Silva, A. Achieving supply chain resilience: the role of procurement. Supply Chain Manag. An Int. J. 2014, 19, 626-642. [CrossRef]

15. Tukamuhabwa, B.R.; Stevenson, M.; Busby, J.; Zorzini, M. Supply chain resilience: definition, review and theoretical foundations for further study. Int. J. Prod. Res. 2015, 53, 5592-5623. [CrossRef]

16. Rice, J.B., Jr.; Caniato, F. Building a secure and resilient supply network. Supply Chain Manag. An Int. J. 2003, 7, 22-30. [CrossRef]

17. Friday, D.; Ryan, S.; Sridharan, R.; Collins, D. Collaborative risk management: A systematic literature review. Int. J. Phys. Distrib. Logist. Manag. 2018, 48, 231-253. [CrossRef]

18. Stone, J.; Rahimifard, S. Resilience in agri-food supply chains: A critical analysis of the literature and synthesis of a novel framework. Supply Chain Manag. An Int. J. 2018, 23, 207-238. [CrossRef]

19. Dania, W.A.P.; Xing, K.; Amer, Y. Collaboration behavioural factors for sustainable agri-food supply chains: A systematic review. J. Clean. Prod. 2018, 186, 851-864. [CrossRef]

20. Ruiz-Benitez, R.; López, C.; Real, J.C. Environmental benefits of lean, green and resilient supply chain management: The case of the aerospace sector. J. Clean. Prod. 2018, 167, 850-862. [CrossRef]

21. Dubey, R.; Gunasekaran, A.; Papadopoulos, T.; Childe, S.J.; Shibin, K.T.; Wamba, S.F. Sustainable supply chain management: framework and further research directions. J. Clean. Prod. 2017, 142, 1119-1130. [CrossRef]

22. Tranfield, D.; Denyer, D.; Smart, P. Towards a Methodology for Developing Evidence-Informed Management Knowledge by Means of Systematic Review. Br. J. Manag. 2003, 14, 207-222. [CrossRef]

23. Bhosale, V.A.; Kant, R. An integrated ISM fuzzy MICMAC approach for modelling the supply chain knowledge flow enablers. Int. J. Prod. Res. 2016, 54, 7374-7399. [CrossRef]

24. Webster, J.; Watson, R.T. Analyzing the past to prepare for the future: Writing a literature review. MIS Q. 2002, 26, xiii-xxiii. [CrossRef]

25. Kitchenham, B. Procedures for performing systematic reviews. Keele, UK, Keele Univ. 2004, 33, 1-26.

26. Alexander, A.; Walker, H.; Naim, M. Decision theory in sustainable supply chain management: A literature review. Supply Chain Manag. An Int. J. 2014, 19, 504-522. [CrossRef]

27. Busalim, A.H.; Hussin, A.R.C. Understanding social commerce: A systematic literature review and directions for further research. Int. J. Inf. Manage. 2016, 36, 1075-1088. [CrossRef]

28. Brusset, X.; Teller, C. Supply chain capabilities, risks, and resilience. Int. J. Prod. Econ. 2017, 184, 59-68. [CrossRef]

29. Malone, D.W. An introduction to the application of interpretive structural modeling. Proc. IEEE 1975, 63, 397-404. [CrossRef]

30. Wu, W.; Yang, C.; Chang, J.-C.; Château, P.; Chang, Y.-C. Risk assessment by integrating interpretive structural modeling and Bayesian network, case of offshore pipeline project. Reliab. Eng. Syst. Saf. 2015, 142, 515-524. [CrossRef]

31. Faisal, M.N.; Banwet, D.K.; Shankar, R. Information risks management in supply chains: An assessment and mitigation framework. J. Enterp. Inf. Manag. 2007, 20, 677-699. [CrossRef]

32. Govindan, K.; Azevedo, S.G.; Carvalho, H.; Cruz-Machado, V. Lean, green and resilient practices influence on supply chain performance: interpretive structural modeling approach. Int. J. Environ. Sci. Technol. 2015, 12, 15-34. [CrossRef]

33. Diabat, A.; Govindan, K.; Panicker, V.V. Supply chain risk management and its mitigation in a food industry. Int. J. Prod. Res. 2012, 50, 3039-3050. [CrossRef]

34. Soni, U.; Jain, V.; Kumar, S. Measuring supply chain resilience using a deterministic modeling approach. Comput. Ind. Eng. 2014, 74, 11-25. [CrossRef]

35. Brandon-Jones, E.; Squire, B.; Autry, C.W.; Petersen, K.J. A contingent resource-based perspective of supply chain resilience and robustness. J. Supply Chain Manag. 2014, 50, 55-73. [CrossRef] 
36. Leat, P.; Revoredo-Giha, C. Risk and resilience in agri-food supply chains: The case of the ASDA PorkLink supply chain in Scotland. Supply Chain Manag. An Int. J. 2013, 18, 219-231. [CrossRef]

37. Blackhurst, J.; Dunn, K.S.; Craighead, C.W. An empirically derived framework of global supply resiliency. J. Bus. Logist. 2011, 32, 374-391. [CrossRef]

38. Khan, O.; Christopher, M.; Creazza, A. Aligning product design with the supply chain: A case study. Supply Chain Manag. An Int. J. 2012, 17, 323-336. [CrossRef]

39. Day, J.M. Fostering emergent resilience: The complex adaptive supply network of disaster relief. Int. J. Prod. Res. 2014, 52, 1970-1988. [CrossRef]

40. Pereira, J.V. The new supply chain's frontier: Information management. Int. J. Inf. Manag. 2009, 29, 372-379. [CrossRef]

41. Boyes, H. Cybersecurity and Cyber-Resilient Supply Chains. Technol. Innov. Manag. Rev. 2015, 5, $28-34$. [CrossRef]

42. Golgeci, I.; Ponomarov, S.Y. Does firm innovativeness enable effective responses to supply chain disruptions? An empirical study. Supply Chain Manag. An Int. J. 2013, 18, 604-617. [CrossRef]

43. Stecke, K.E.; Kumar, S. Sources of supply chain disruptions, factors that breed vulnerability, and mitigating strategies. J. Mark. Channels 2009, 16, 193-226. [CrossRef]

44. Johnson, N.; Elliott, D.; Drake, P.; Johnson, N.; Elliott, D.; Drake, P.; Johnson, N. Exploring the role of social capital in facilitating supply chain resilience. Supply Chain Manag. An Int. J. 2013, 18, 324-336. [CrossRef]

45. Matsuo, H. Implications of the Tohoku earthquake for Toyota's coordination mechanism: Supply chain disruption of automotive semiconductors. Int. J. Prod. Econ. 2015, 161, 217-227. [CrossRef]

46. Purvis, L.; Spall, S.; Naim, M.; Spiegler, V. Developing a resilient supply chain strategy during 'boom' and 'bust'. Prod. Plan. Control 2016, 7287. [CrossRef]

47. Rajesh, R.; Ravi, V. Supplier selection in resilient supply chains: A grey relational analysis approach. J. Clean. Prod. 2015, 86, 343-359. [CrossRef]

48. Baker, P. Designing distribution centres for agile supply chains. Intenational J. Logist. 2006, 9, $207-221$. [CrossRef]

49. Blome, C.; Schoenherr, T.; Eckstein, D. The impact of knowledge transfer and complexity on supply chain flexibility: A knowledge-based view. Int. J. Prod. Econ. 2014, 147, 307-316. [CrossRef]

50. Gligor, D.M.; Autry, C.W. The Role of Personal Ralationships in Facilitating Supply Chain Communications: A Qualitative Study. J. Supply Chain Manag. 2012, 48, 24-43. [CrossRef]

51. Higgins, A.J.; Miller, C.J.; Archer, A.A.; Ton, T.; Fletcher, C.S.; McAllister, R.R.J. Challenges of operations research practice in agricultural value chains. J. Oper. Res. Soc. 2010, 61, 964-973. [CrossRef]

52. Klibi, W.; Martel, A.; Guitouni, A. The design of robust value-creating supply chain networks: A critical review. Eur. J. Oper. Res. 2010, 203, 283-293. [CrossRef]

53. Borgatti, S.P.; Li, X. On Social Network Analysis in a Supply Chain Context. J. Supply Chain Manag. 2009, 1-17.

54. Lin, C.T.; Chiu, H.; Chu, P.Y. Agility index in the supply chain. Int. J. Prod. Econ. 2006, 100, $285-299$. [CrossRef]

55. Manuj, I.; Mentzer, J.T. Global supply chain risk management strategies. Int. J. Phys. Distrib. Logist. Manag. 2008, 38, 192-223. [CrossRef]

56. Melnyk, S.A.; Davis, E.W.; Spekman, R.E.; Sandor, J. Outcome-Driven Supply Chains. MIT Sloan Manag. Rev. 2010.

57. Narasimhan, R.; Swink, M.; Kim, S.W. Disentangling leanness and agility: An empirical investigation. J. Oper. Manag. 2006, 24, 440-457. [CrossRef]

58. Purvis, L.; Gosling, J.; Naim, M.M. The development of a lean, agile and leagile supply network taxonomy based on differing types of flexibility. Int. J. Prod. Econ. 2014, 151, 100-111. [CrossRef]

59. Stevenson, M.; Spring, M. Flexibility from a Supply Chain Perspective: Definition and Review; Emerald Publishing Limited: Bingley, UK, 2007; Volume 27, ISBN 0144357071075.

60. Swafford, P.M.; Ghosh, S.; Murthy, N. The antecedents of supply chain agility of a firm: Scale development and model testing. J. Oper. Manag. 2006, 24, 170-188. [CrossRef]

61. Wieland, A.; Wallenburg, C.M.; Wieland, A. The influence of relational competencies on supply chain resilience: A relational view. Int. J. Phys. Distrib. Logist. Manag. 2013, 43, 300-320. [CrossRef]

62. Wieland, A. Selecting the right supply chain based on risks. J. Manuf. Technol. Manag. 2013, 24, 652-668. [CrossRef] 
63. Li, X.; Goldsby, T.J.; Holsapple, C.W. Supply chain agility: scale development. Int. J. Logist. Manag. 2009, 20, 408-424. [CrossRef]

64. Zsidisin, G.A.; Wagner, S.M. Do perceptions become reality? The moderating role of supply chain resiliency on disruption occurrence. J. Bus. Logist. 2010, 31, 1-20. [CrossRef]

65. Mandal, S.; Sarathy, R.; Korasiga, V.R.; Bhattacharya, S.; Dastidar, S.G. Achieving supply chain resilience. Int. J. Disaster Resil. Built Environ. 2016, 7, 544-562. [CrossRef]

66. Carvalho, H.; Azevedo, S.G.; Cruz-Machado, V. Agile and resilient approaches to supply chain management: Influence on performance and competitiveness. Logist. Res. 2012, 4, 49-62. [CrossRef]

67. Berle, Ø.; Norstad, I.; Asbjørnslett, B.E. Optimization, risk assessment and resilience in LNG transportation systems. Supply Chain Manag. An Int. J. 2013, 18, 253-264. [CrossRef]

68. Tang, C.; Tomlin, B. The power of flexibility for mitigating supply chain risks. Int. J. Prod. Econ. 2008, 116, 12-27. [CrossRef]

69. Chiang, C.; Kocabasoglu-Hillmer, C.; Suresh, N. An empirical investigation of the impact of strategic sourcing and flexibility on firm's supply chain agility. Int. J. Oper. Prod. Manag. 2012, 32, 49-78. [CrossRef]

70. Christopher, M.; Holweg, M. "Supply Chain 2.0": managing supply chains in the era of turbulence. Int. J. Phys. Distrib. Logist. Manag. 2011, 41, 63-82. [CrossRef]

71. Schmitt, A.J.; Singh, M. A quantitative analysis of disruption risk in a multi-echelon supply chain. Int. J. Prod. Econ. 2012, 139, 22-32. [CrossRef]

72. Durach, C.F.; Wieland, A.; Machuca, J.A.D. Antecedents and dimensions of supply chain robustness: A systematic literature review. Int. J. Phys. Distrib. Logist. Manag. 2015, 45, 118-137. [CrossRef]

73. Christopher, M.; Mena, C.; Khan, O.; Yurt, O. Approaches to managing global sourcing risk. Supply Chain Manag. An Int. J. 2011, 16, 67-81. [CrossRef]

74. Peck, H. Drivers of Supply Chain Vulnerability: An Integrated Framework; Emerald Publishing Limited: Bingley, UK, 2005; Volume 35, ISBN 1040359019.

75. Wei, H.-L.; Wang, E.T.G. The strategic value of supply chain visibility: increasing the ability to reconfigure. Eur. J. Inf. Syst. 2010, 19, 238-249. [CrossRef]

76. Francis, V. Supply chain visibility: lost in translation? Supply Chain Manag. An Int. J. 2008, 13, $180-184$. [CrossRef]

77. Li, X.; Wu, Q.; Holsapple, C.W.; Goldsby, T. An empirical examination of firm financial performance along dimensions of supply chain resilience. Manag. Res. Rev. 2017, 40, 254-269. [CrossRef]

78. Tachizawa, E.M.; Gimenez, C. Supply flexibility strategies in Spanish firms: Results from a survey. Int. J. Prod. Econ. 2010, 124, 214-224. [CrossRef]

79. Carvalho, H.; Azevedo, S.G.; Machado, V.C. Supply chain management resilience: A theory building approach. Int. J. Supply Chain Oper. Resil. 2014, 1, 3. [CrossRef]

80. Ratick, S.; Meacham, B.; Aoyama, Y. Chain Disaster Resilience. Growth Change 2008, 39, 642-666. [CrossRef]

81. Fiksel, J.; Polyviou, M.; Croxton, K.L.; Pettit, T.J. From Risk to Resilience: Learning to Deal With Disruption. MIT Sloan Manag. Rev. 2015, 56, 79-86. [CrossRef]

82. Cao, M.; Zhang, Q. Supply chain collaboration: impact on collaborative advantage and firm performance. J. Oper. Manag. 2011, 29, 163-180. [CrossRef]

83. Danese, P.; Romano, P. Supply chain integration and efficiency performance: A study on the interactions between customer and supplier integration. Supply Chain Manag. An Int. J. 2011, 16, 220-230. [CrossRef]

84. Scholten, K.; Schilder, S. The role of collaboration in supply chain resilience. Supply Chain Manag. An Int. J. 2015, 20, 471-484. [CrossRef]

85. Simangunsong, E.; Hendry, L.C.; Stevenson, M. Supply-chain uncertainty: A review and theoretical foundation for future research. Int. J. Prod. Res. 2012, 50, 4493-4523. [CrossRef]

86. Spiegler, V.L.M.; Naim, M.M.; Wikner, J. A control engineering approach to the assessment of supply chain resilience. Int. J. Prod. Res. 2012, 50, 6162-6187. [CrossRef]

87. Yi, C.Y.; Ngai, E.W.T.; Moon, K. Supply chain flexibility in an uncertain environment: exploratory findings from five case studies. Supply Chain Manag. An Int. J. 2011, 16, 271-283. [CrossRef]

88. Bakshi, N.; Kleindorfer, P. Co-opetition and Investment for Supply-Chain Resilience. Prod. Oper. Manag. 2009, 18, 583-603. [CrossRef]

89. Voss, M.D.; Williams, Z. Public-Private Partnerships and Supply Chain Security: C- TPAT as an Indicator of Relational Security. J. Bus. Logist. 2013, 34, 320-334. [CrossRef] 
90. Sáenz, M.J.; Revilla, E.; Saenz, M.J. Creating More Resilient Supply Chains. MIT Sloan Manag. Rev. 2014, 55, 22-24. [CrossRef]

91. Fawcett, S.E.; Osterhaus, P.; Magnan, G.M.; Brau, J.C.; McCarter, M.W. Information sharing and supply chain performance: the role of connectivity and willingness. Supply Chain Manag. An Int. J. 2007, 12, 358-368. [CrossRef]

92. Sheu, C.; Rebecca Yen, H.; Chae, B. Determinants of supplier-retailer collaboration: Evidence from an international study. Int. J. Oper. Prod. Manag. 2006, 26, 24-49. [CrossRef]

93. Gligor, D.M.; Holcomb, M. The road to supply chain agility: An RBV perspective on the role of logistics capabilities. Int. J. Logist. Manag. 2014, 25, 160-179. [CrossRef]

94. Boone, C.A.; Craighead, C.W.; Hanna, J.B.; Nair, A. Implementation of a system approach for enhanced supply chain continuity and resiliency: A longitudinal study. J. Bus. Logist. 2013, 34, 222-235. [CrossRef]

95. Ivanov, D.; Sokolov, B.; Dolgui, A. The ripple effect in supply chains: trade-off 'efficiency-flexibility-resilience' in disruption management. Int. J. Prod. Res. 2014, 52, 2154-2172. [CrossRef]

96. Urciuoli, L. Cyber-Resilience: A Strategic Approach for Supply Chain Management. Technol. Innov. Manag. Rev. 2015, 13-19. [CrossRef]

97. Urciuoli, L.; Mohanty, S.; Hintsa, J.; Gerine Boekesteijn, E. The resilience of energy supply chains: A multiple case study approach on oil and gas supply chains to Europe. Supply Chain Manag. An Int. J. 2014, 19, 46-63. [CrossRef]

98. Barratt, M.; Oke, A. Antecedents of supply chain visibility in retail supply chains: A resource-based theory perspective. J. Oper. Manag. 2007, 25, 1217-1233. [CrossRef]

99. Boyson, S.; Corsi, T.; Verbraeck, A. The e-supply chain portal: A core business model. Transp. Res. Part E Logist. Transp. Rev. 2003, 39, 175-192. [CrossRef]

100. Carvalho, H.; Barroso, A.P.; MacHado, V.H.; Azevedo, S.; Cruz-Machado, V. Supply chain redesign for resilience using simulation. Comput. Ind. Eng. 2012, 62, 329-341. [CrossRef]

101. Tang, C. Robust strategies for mitigating supply chain disruptions. Int. J. Logist. 2006, 9, 33-45. [CrossRef]

102. Zacharia, Z.G.; Mentzer, J.T. Logistics Salience in a Changing Environment. J. Bus. Logist. 2004, 25, 187-210. [CrossRef]

103. Li, G.; Lin, Y.; Wang, S.; Yan, H. Enhancing agility by timely sharing of supply information. Supply Chain Manag. An Int. J. 2006, 11, 425-435. [CrossRef]

104. Stewart, G.T.; Kolluru, R.; Smith, M. Leveraging public-private partnerships to improve community resilience in times of disaster. Int. J. Phys. Distrib. Logist. Manag. 2009, 39, 343-364. [CrossRef]

105. Eltantawy, R.A. The role of supply management resilience in attaining ambidexterity: A dynamic capabilities approach. J. Bus. Ind. Mark. 2016, 31, 123-134. [CrossRef]

106. Ismail, H.S.; Poolton, J.; Sharifi, H. The role of agile strategic capabilities in achieving resilience in manufacturing-based small companies. Int. J. Prod. Res. 2011, 49, 5469-5487. [CrossRef]

107. Sprecher, B.; Daigo, I.; Murakami, S.; Kleijn, R.; Vos, M.; Kramer, G.J. Framework for resilience in material supply chains, with a case study from the 2010 rare earth crisis. Environ. Sci. Technol. 2015, 49, 6740-6750. [CrossRef] [PubMed]

108. Ambulkar, S.; Blackhurst, J.; Grawe, S. Firm's resilience to supply chain disruptions: Scale development and empirical examination. J. Oper. Manag. 2015, 33-34, 111-122. [CrossRef]

109. Pagell, M. Understanding the factors that enable and inhibit the integration of operations, purchasing and logistics. J. Oper. Manag. 2004, 22, 459-487. [CrossRef]

110. Rajaguru, R.; Matanda, M.J. Effects of inter-organizational compatibility on supply chain capabilities: Exploring the mediating role of inter-organizational information systems (IOIS) integration. Ind. Mark. Manag. 2013, 42, 620-632. [CrossRef]

111. Williams, Z.; Ponder, N.; Autry, C.W. Supply chain security culture: Measure development and validation. Int. J. Logist. Manag. 2009, 20, 243-260. [CrossRef]

112. Blos, M.F.; Wee, H.-M.; Yang, J. Analysing the external supply chain risk driver competitiveness: A risk mitigation framework and business continuity plan. J. Bus. Contin. Emer. Plan. 2010, 4, 368-374. [PubMed]

113. VanVactor, J.D. Cognizant healthcare logistics management: Ensuring resilience during crisis. Int. J. Disaster Resil. Built Environ. 2011, 2, 245-255. [CrossRef]

114. Davis, A. Building Cyber-Resilience into Supply Chains. Technol. Innov. Manag. Rev. 2015, 19-27. [CrossRef] 
115. Machado, S.M.; Paiva, E.L.; da Silva, E.M. Counterfeiting: Addressing mitigation and resilience in supply chains. Int. J. Phys. Distrib. Logist. Manag. 2018, 48, 139-163. [CrossRef]

116. Kannan, D. Role of multiple stakeholders and the critical success factor theory for the sustainable supplier selection process. Int. J. Prod. Econ. 2018, 195, 391-418. [CrossRef]

117. Chopra, S.; Sodhi, M.S. Managing risk to avoid supply-chain breakdown. MIT Sloan Manag. Rev. 2004.

118. Pomponi, F.; Fratocchi, L.; Tafuri, S.R.; Rossi Tafuri, S. Trust development and horizontal collaboration in logistics: A theory based evolutionary framework. Supply Chain Manag. An Int. J. 2015, 20, 83-97. [CrossRef]

119. Wu, L.Y.; Chen, P.Y.; Chen, K.Y. Why does loyalty-cooperation behavior vary over buyer-seller relationship? J. Bus. Res. 2015, 68, 2322-2329. [CrossRef]

120. Wu, T.; Huang, S.M.; Blackhurst, J.; Zhang, X.L.; Wang, S.S. Supply Chain Risk Management: An Agent-Based Simulation to Study the Impact of Retail Stockouts. Ieee Trans. Eng. Manag. 2013, 60, 676-686. [CrossRef]

121. Koschmann, M.A.; Kuhn, T.R.; Pfarrer, M.D. A communicative framework of value in cross-sector partnerships. Acad. Manag. Rev. 2012, 37, 332-354. [CrossRef]

122. Erker, S.; Stangl, R.; Stoeglehner, G. Resilience in the light of energy crises - Part I a framework to conceptualise regional energy resilience. J. Clean. Prod. 2017, 164, 420-433. [CrossRef]

123. Rostamzadeh, R.; Ghorabaee, M.K.; Govindan, K.; Esmaeili, A.; Nobar, H.B.K. Evaluation of sustainable supply chain risk management using an integrated fuzzy TOPSIS- CRITIC approach. J. Clean. Prod. 2018, 175, 651-669. [CrossRef]

124. Morais, D.O.C.; Silvestre, B.S. Advancing Social Sustainability in Supply Chain Management: Lessons from Multiple Case Studies in an Emerging Economy. J. Clean. Prod. 2018, 199, 222-235. [CrossRef]

125. Gligor, D.M.; Esmark, C.L. Supply chain friends: The good, the bad, and the ugly. Bus. Horiz. 2015, 58, 517-525. [CrossRef]

(C) 2019 by the authors. Licensee MDPI, Basel, Switzerland. This article is an open access article distributed under the terms and conditions of the Creative Commons Attribution (CC BY) license (http:// creativecommons.org/licenses/by/4.0/). 